\title{
The Spectral Web of stationary plasma equilibria: II. Internal modes
}

\author{
J.P. Goedbloed \\ DIFFER - Dutch Institute for Fundamental Energy Research, \\ De Zaale 20, 5612 AJ Eindhoven, the Netherlands
}

(Dated: February 5, 2018)

\begin{abstract}
The new method of the Spectral Web to calculate the spectrum of waves and instabilities of plasma equilibria with sizeable flows, developed in the preceding Paper $\mathrm{I}^{1}$ is applied to a collection of classical magnetohydrodynamic (MHD) instabilities operating in cylindrical plasmas with shear flow or rotation. After a review of the basic concepts of the complementary energy giving the solution path and the conjugate path, which together constitute the Spectral Web, the cylindrical model is presented and the spectral equations are derived. The first example concerns the internal kink instabilities of a cylindrical force-free magnetic field of constant $\alpha$ subjected to a parabolic shear flow profile. The old stability diagram and the associated growth rate calculations for static equilibria are replaced by a new intricate stability diagram and associated complex growth rates for the stationary model. The power of the Spectral Web method is demonstrated by showing that the two associated paths in the complex $\omega$-plane nearly automatically guide to the new class of Global Alfvén Instabilities (GAIs) of the force-free configuration, that would have been very hard to predict by other methods. The second example concerns the Rayleigh-Taylor instability of a rotating theta-pinch. The old literature is revisited and shown to suffer from inconsistencies that are remedied. The most global $n=1$ instability and a cluster sequence of more local but much more unstable $n=2,3, \ldots \infty$ modes are located on separate solution paths in the hydrodynamic (HD) version of the instability, whereas they merge in the MHD version. The Spectral Web offers visual demonstration of the central position the HD flow continuum and of the MHD Alfvén and slow magneto-sonic continua in the respective spectra by connecting the discrete modes in the complex plane by physically meaningful curves towards the continua. The third example concerns the magneto-rotational instability (MRI) thought to be operating in accretion disks about black holes. The sequence $n=1,2, \ldots$ of unstable MRIs is located on one continuous solution path, but also on infinitely many separate loops ('pancakes') of the conjugate path with just one MRI on each of them. For narrow accretion disks, those sequences are connected with the slow magneto-sonic continuum, which is far away though from the marginal stability transition. In this case, the Spectral Web method is the first to effectively incorporate the MRIs into the general MHD spectral theory of equilibria with background flows. Together, the three examples provide compelling evidence of the computational power of the Spectral Web Method.
\end{abstract}

PACS numbers: 52.30.Cv, 52.30.-q, 52.35.Bj, 47.65.-d 


\section{INTRODUCTION}

In the preceding paper, ${ }^{1}$ henceforth referred to as Paper I, a new method was introduced to obtain the ideal magnetohydrodynamic (MHD) spectrum of waves and instabilities of stationary equilibria. This involves the construction of what was called the Spectral Web, consisting of a dual set of curves in the complex $\omega$-plane, the solution path and the conjugate path, with the eigenvalues on the intersections. In this paper, the Spectral Web method will be applied to the computation of the MHD spectra for the standard 'diffuse linear pinch', ${ }^{2}$ i.e. a cylindrical plasma of infinite length or of finite length (a so called periodic cylinder, frequently exploited to model toroidal tokamak plasmas). We will show how the Spectral Web for stationary, in particular rotating, plasmas drastically changes our perspective on the classical instabilities of internal and external MHD modes of laboratory and astrophysical plasmas. The method is extended to external kink modes and resistive wall modes in a forthcoming paper. The present paper is restricted to cylindrical geometry, but the Spectral Web method is equally valid for toroidal plasmas, as was shown in Paper I.

Recall that the appropriate starting point for the study of MHD waves and instabilities of stationary equilibria is the spectral differential equation of Frieman and Rotenberg ${ }^{3}$ for normal modes $\exp (-\mathrm{i} \omega t)$ of the plasma displacement vector field $\boldsymbol{\xi}$ :

$$
\mathbf{G}(\boldsymbol{\xi})-2 \rho \omega U \boldsymbol{\xi}+\rho \omega^{2} \boldsymbol{\xi}=0 .
$$

Here, $\mathbf{G}(\boldsymbol{\xi}) \equiv \mathbf{F}(\boldsymbol{\xi})+\nabla \cdot(\boldsymbol{\xi} \rho \mathbf{v} \cdot \nabla \mathbf{v})-\rho(\mathbf{v} \cdot \nabla)^{2} \boldsymbol{\xi}$ is the force operator for stationary equilibria, generalizing the well-known expression $\mathbf{F}(\boldsymbol{\xi})$ for static equilibria, ${ }^{4}$ and $U \equiv-\mathrm{iv} \cdot \nabla$ is the gradient operator projected onto the equilibrium velocity. Applying closed-system boundary conditions (BCs) on $\boldsymbol{\xi}$, Eq. (1) becomes a quadratic eigenvalue problem in terms of the two operators $\mathbf{G}$ and $U$, which are both self-adjoint. Hence, the two associated quadratic forms

$$
W \equiv-\frac{1}{2} \int \boldsymbol{\xi}^{*} \cdot \mathbf{G}(\boldsymbol{\xi}) d V, \text { and } \quad V \equiv \frac{1}{2} \int \rho \boldsymbol{\xi}^{*} \cdot U \boldsymbol{\xi} d V
$$

are real for solutions satisfying those BCs. Introducing the normalization $I \equiv \frac{1}{2} \int \rho|\boldsymbol{\xi}|^{2} d V$, a quadratic equation for the complex eigenvalue $\omega \equiv \sigma+\mathrm{i} \nu$ is obtained:

$$
\omega^{2}-2 \bar{V} \omega-\bar{W}=0, \quad \bar{V} \equiv V / I, \quad \bar{W} \equiv W / I,
$$

where $\bar{V}$ and $\bar{W}$ are the solution averages of the Doppler-Coriolis shift and of the potential energy of the perturbations, respectively. For instabilities with real frequency $\sigma$ and growth rate $\nu$, the solutions of this quadratic equation may be written as

$$
\sigma=\bar{V}, \quad \nu= \pm\left(-\bar{W}-\bar{V}^{2}\right)^{1 / 2}
$$

However, these expressions cannot be evaluated a priori. In the standard approach, they can only be computed a posteriori, i.e. by substituting the final solution of the spectral equation (1) consisting of the eigenvalue eigenfunction pair $\{\omega, \boldsymbol{\xi}(\mathbf{r} ; \omega)\}$.

In Ref. 5, a new method of solving the quadratic eigenvalue problem (1) + BCs was developed based on the fact that the operator $U$ is actually self-adjoint irrespective of whether $\boldsymbol{\xi}$ satisfies the BCs or not. Hence, $\bar{V}$ is real throughout the complex $\omega$-plane for any solution of Eq. (1), not necessarily an eigenfunction. In particular, one may compute the value of 
$\bar{V}$ for arbitrary $\omega$ by 'shooting' from the magnetic axis with solutions of Eq. (1) that only satisfy the $\mathrm{BC}$ there. For a given value $\nu=\nu_{0}$, the resulting nonlinear algebraic equation,

$$
\sigma-\bar{V}\left[\boldsymbol{\xi}\left(\mathbf{r} ; \sigma+\mathrm{i} \nu_{0}\right)\right]=0 \quad \text { (solution path) }
$$

is solved by straightforward iteration on the zeros, giving one or more solutions $\sigma=\sigma_{0}\left(\nu_{0}\right)$. The collection of all these points $\left\{\sigma_{0}\left(\nu_{0}\right)+\mathrm{i} \nu_{0}\right\}$ of the $\omega$-plane, where the Doppler-Coriolis shift of the real part of the frequency vanishes, was called the solution path. The actual eigenvalues were obtained by a second iteration, along the solution path, to also satisfy the remaining, outer, BC. Thus, the solution path may be considered as the counterpart for stationary equilibria of the imaginary axis $(\sigma=0)$, on which the eigenvalues are constrained to lie for static equilibria.

In Paper I, the solution path method was significantly improved by computing the Spectral Web. This involves considering the effect of the open boundary on the quadratic form $W$, which then becomes complex:

$$
W=W_{\text {sym }}+W_{\text {com }}
$$

Here, $W_{\text {sym }}$ is the usual symmetric (real) volume integral of the plasma potential energy, whereas $W_{\text {com }}$ is the complex surface integral representing the energy that would have to be injected into of extracted from the plasma through the open boundary in order to maintain the time-dependence of the system at $\exp (-\mathrm{i} \omega t)$ with a particular value of $\omega$. The eigenvalues are then obtained by computing the zeros of that complementary energy, where the potential energy becomes real again:

$$
W_{\text {com }}[\boldsymbol{\xi}(\mathbf{r} ; \omega)]=0 \Rightarrow\left\{\begin{array}{ll}
\operatorname{Im}\left(W_{\text {com }}\right)=0 \quad \text { (solution path) } \\
\operatorname{Re}\left(W_{\text {com }}\right)=0 \quad \text { (conjugate path) }
\end{array} .\right.
$$

This involves computing two paths, viz. the mentioned solution path and another path, called the conjugate path. The ideal MHD spectrum of unstable modes of stationary equilibria is then found by constructing the Spectral Web consisting of the superposition of the curves of the solution path and the conjugate path in the complex $\omega$-plane, whereas the eigenvalues are located at the intersections of those curves.

\section{STATIONARY CYLINDRICAL PLASMAS}

\section{A. Model equilibria}

The most basic model for confined plasmas is the circular cylinder model with a cylindrically symmetric magnetic field and velocity with a longitudinal $(z)$ and an azimuthal $(\theta)$ component. For the equilibrium of such translating-rotating plasmas, just one restriction needs to be observed on the radial dependence of the density $\rho$, the pressure $p$, the magnetic field components $B_{\theta}, B_{z}$, and the velocity components $v_{\theta}, v_{z}$, viz.:

$$
\left(p+\frac{1}{2} B^{2}\right)^{\prime}=\left(\rho v_{\theta}^{2}-B_{\theta}^{2}\right) / r-\rho \Phi_{\mathrm{gr}}^{\prime}, \quad \Phi_{\mathrm{gr}} \approx-G M_{*} / r,
$$

where the prime indicates differentiation with respect to $r$. The gravitational potential $\Phi_{\mathrm{gr}}$ of an external compact object of mass $M_{*}$ is included for the analysis of the magneto-rotational 
instabilities of thin accretion disks in the cylindrical approximation (Section V). Of course, this contribution is dropped for laboratory plasmas.

The circular cylinder symmetry permits to express the displacement vector in terms of a sum of uncoupled Fourier harmonics of the form

$$
\boldsymbol{\xi}(r, \theta, z, t)=\left[\xi_{r}(r) \mathbf{e}_{r}+\xi_{\theta}(r) \mathbf{e}_{\theta}+\xi_{z}(r) \mathbf{e}_{z}\right] \mathrm{e}^{\mathrm{i}(m \theta+k z-\omega t)},
$$

where $m$ is an integer and $k$ is a real number. We will consider four kinds of cylindrical equilibrium models having different restrictions on the wave number $k$, viz.:

(a) standard infinite cylinder: $-\infty<k<\infty$;

(b) 'straight' tokamak, a periodic cylinder of length $L=2 \pi R_{0}$ modeling a toroidal plasma of major radius $R_{0}: k=n / R_{0}$ with integer $n$;

(c) rotating jet, a cylinder of length $L$ with fixed ends: $k=n \pi / L$ with integer $n$;

(d) thin accretion disk, an annular cylinder with $\Delta r \equiv r_{2}-r_{1}$ and small $L=\Delta z \ll \Delta r$, so that the approximation (8)(b) for the gravitational potential is valid: $k \Delta z \gg 1$.

For model (a), the normalized (inverse) pitch of the magnetic field lines is expressed by $\mu \equiv B_{\theta} /\left(r B_{z}\right)$. For the models (b) and (c), it is expressed in terms of the tokamak safety factor, $q \equiv\left(\mu R_{0}\right)^{-1} \equiv r B_{z} /\left(R_{0} B_{\theta}\right)=4 \pi r^{2} B_{z} /\left(L I_{z}\right)$, where $I_{z}$ is the total longitudinal current flowing in the plasma, so that the parallel gradient operator becomes

$$
F \equiv-\mathrm{i} \mathbf{B} \cdot \nabla=m B_{\theta} / r+k B_{z}= \begin{cases}(k+\mu m) B_{z} & \text { (infinite cylinder) } \\ (m / q+n) B_{z} / R_{0} & \text { (tokamak) } \\ \left(m / q+\frac{1}{2} n\right) 2 \pi B_{z} / L & \text { (jet) }\end{cases}
$$

We also define the perpendicular gradient operator, $G \equiv-\mathrm{i}(\mathbf{B} \times \mathbf{n}) \cdot \nabla=m B_{z} / r-k B_{\theta}$, for later use. For plasma surrounded by vacuum, the Kruskal-Shafranov condition for the absence of a rational magnetic surface $F=0$ in the vacuum, i.e. stability with respect to the $m=n=1$ external kink mode of a static plasma, then becomes:

$$
q(r=a)>1 \quad(\text { tokamak }), \quad q(r=a)>2 \quad(\text { jet }) .
$$

In normal tokamak operation, this limit is always observed. In astrophysical jets, this limit usually appears to be violated so that it becomes imperative to consider the modifications caused by the equilibrium background velocities $v_{\theta}$ and $v_{z}$. Since the present paper is restricted to internal modes, this topic is relegated to future investigations.

\section{B. Spectral differential equations}

To implement the Spectral Web method, we first need to solve the spectral differential equation. For translating-rotating cylindrical plasma equilibria, this becomes a second order ordinary differential equation (ODE) for the radial component $\chi \equiv r \xi_{r}$ of the plasma displacement, obtained from the Frieman-Rotenberg spectral equation (1) in the cylindrical 
limit, ${ }^{6,7}$ for astrophysical applications extended with the contributions of the gravitational potential. ${ }^{8}$ We here exploit the representation given in Eq. (13.107) of Ref. 9:

$$
\frac{d}{d r}\left(\frac{N}{D} \frac{d \chi}{d r}\right)+\left[A+\frac{B}{D}+\left(\frac{C}{D}\right)^{\prime}\right] \chi=0,
$$

where the coefficients are defined below. This ODE is to be solved subject to the BCs

$$
\chi(0)=0, \quad \chi(a)=0,
$$

where the second one is due to a rigid wall at $r=a$. For the annular model $(\mathrm{d})$, the positions $r=0$ and $r=a$ are to be replaced by $r=r_{1}$ and $r=r_{2}$, of course not to be considered as positions of rigid walls but as defining the region of localization of the eigenfunctions.

For stationary equilibria, the coefficients of the spectral differential equations not only involve the gradient operator projected onto the magnetic field, $F$, defined in Eq. (10), but also the gradient operator projected onto the velocity field, $U \equiv-\mathrm{iv} \cdot \nabla$ (connected to the solution-averaged Doppler-Coriolis shift defined in Eq. (22) below). The Doppler part of that operator appears in the coefficients through the local Doppler-shifted frequency

$$
\widetilde{\omega}(r) \equiv \omega-\Omega_{0}(r), \quad \Omega_{0}(r) \equiv m v_{\theta}(r) / r+k v_{z}(r) .
$$

The singularity coefficients $N$ and $D$ of the ODE (12) are defined by

$$
\begin{array}{rlrl}
N \equiv \frac{1}{r} \widetilde{A} \widetilde{S}, & & \widetilde{A} \equiv \rho\left(\widetilde{\omega}^{2}-\omega_{\mathrm{A}}^{2}\right) \equiv \rho\left(\omega-\Omega_{\mathrm{A}}^{+}\right)\left(\omega-\Omega_{\mathrm{A}}^{-}\right), \\
& \widetilde{S} \equiv \rho\left(\gamma p+B^{2}\right)\left(\widetilde{\omega}^{2}-\omega_{\mathrm{S}}^{2}\right) \equiv \rho\left(\gamma p+B^{2}\right)\left(\omega-\Omega_{\mathrm{S}}^{+}\right)\left(\omega-\Omega_{\mathrm{S}}^{-}\right), \\
D \equiv \widetilde{f}_{0} \widetilde{s}_{0}, & & \widetilde{f}_{0} \equiv \rho\left(\widetilde{\omega}^{2}-\omega_{\mathrm{f} 0}^{2}\right) \equiv \rho\left(\omega-\Omega_{\mathrm{f} 0}^{+}\right)\left(\omega-\Omega_{\mathrm{f} 0}^{-}\right), \\
& \widetilde{s}_{0} \equiv \rho\left(\widetilde{\omega}^{2}-\omega_{\mathrm{s} 0}^{2}\right) \equiv \rho\left(\omega-\Omega_{\mathrm{s} 0}^{+}\right)\left(\omega-\Omega_{\mathrm{s} 0}^{-}\right) .
\end{array}
$$

An alternative expression for the latter reads: $D \equiv \rho^{2} \widetilde{\omega}^{4}-h^{2} \widetilde{S}$, where $h^{2} \equiv m^{2} / r^{2}+k^{2}$. The expressions $\Omega_{\mathrm{A}}^{ \pm}$and $\Omega_{\mathrm{S}}^{ \pm}$represent the forward/backward $( \pm)$Alfvén and slow genuine singularities associated with the four continuous spectra of stationary equilibria:

$$
\begin{gathered}
\Omega_{\mathrm{A}}^{ \pm}(r) \equiv \Omega_{0}(r) \pm \omega_{\mathrm{A}}(r), \quad \Omega_{\mathrm{S}}^{ \pm}(r) \equiv \Omega_{0}(r) \pm \omega_{\mathrm{S}}(r), \\
\omega_{\mathrm{A}}^{2} \equiv F^{2} / \rho, \quad \omega_{\mathrm{S}}^{2} \equiv\left[\gamma p /\left(\gamma p+B^{2}\right)\right] \omega_{\mathrm{A}}^{2}
\end{gathered}
$$

where $\pm \omega_{\mathrm{A}}$ and $\pm \omega_{\mathrm{S}}$ are the static counterparts. Likewise, the expressions $\Omega_{\mathrm{f} 0}^{ \pm}$and $\Omega_{\mathrm{s} 0}^{ \pm}$represent the forward/backward $( \pm)$ fast and slow apparent singularities of stationary equilibria:

$$
\begin{aligned}
& \Omega_{\mathrm{f} 0}^{ \pm}(r) \equiv \Omega_{0}(r) \pm \omega_{\mathrm{f} 0}(r), \quad \Omega_{\mathrm{s} 0}^{ \pm}(r) \equiv \Omega_{0}(r) \pm \omega_{\mathrm{s} 0}(r) \\
& \omega_{\mathrm{f} 0 / \mathrm{s} 0}^{2} \equiv \frac{1}{2} \omega_{\mathrm{m}}^{2}\left[1 \pm\left(1-4 \omega_{\mathrm{S}}^{2} / \omega_{\mathrm{m}}^{2}\right)^{1 / 2}\right], \quad \omega_{\mathrm{m}}^{2} \equiv h^{2}\left(\gamma p+B^{2}\right) / \rho
\end{aligned}
$$

The expressions of the remaining coefficients $A, B$ and $C$ are given in Appendix A.

For numerical integration, a representation by first order ODEs is to be preferred over a second order one. By means of the Eulerian total pressure perturbation,

$$
\Pi \equiv-\left(N \chi^{\prime}+C \chi\right) / D
$$


the second order ODE (12) may be transformed into the equivalent system of first order ODEs in terms of $\chi$ and $\Pi$ :

$$
N \frac{d}{d r}\left(\begin{array}{c}
\chi \\
\Pi
\end{array}\right)+\left(\begin{array}{cc}
C & D \\
E & -C
\end{array}\right)\left(\begin{array}{l}
\chi \\
\Pi
\end{array}\right)=0
$$

The new coefficient $E$ is related to the coefficients of Eq. (12) by

$$
E \equiv-N(A+B / D)-C^{2} / D
$$

This expression exhibits two significant proportionalities, viz. $N B+C^{2} \sim D$, which guarantees the absence of spurious $D=0$ singularities in the second order formulation, and $D E+C^{2} \sim N$, which guarantees the absence of spurious $N=0$ singularities in the first order formulation. Crucially, the explicit expression for $E$, given in Appendix A, is a polynomial in $\widetilde{\omega}$, just like the other five coefficients.

For real values of $\widetilde{\omega}$, all of the above expressions are real. For complex values of $\widetilde{\omega}$, the expressions need to be split into real and imaginary parts. Writing $\omega \equiv \sigma+\mathrm{i} \nu$, it is noticed that the $r$-dependence of $\widetilde{\omega}(r)=\widetilde{\sigma}(r)+\mathrm{i} \nu$ only resides in the real part: $\widetilde{\sigma}(r) \equiv \sigma-\Omega_{0}(r)$. Thus, for complex values of $\widetilde{\omega}$, Eq. (20) becomes a system of four first order ODEs. Since they are singular at $r=0$, numerical integration requires the expansions of Appendix B. The consequences of real singularities away from the origin are presented in Appendix C.

\section{Spectral Web and alternator}

Since numerical integration of the system of two (for real $\omega$ ) or four (for complex $\omega$ ) first order ODEs (20) starting from the origin is straightforward, the only problem left is to design an iterative procedure for the eigenvalue search in the complex $\omega$-plane such that, eventually, both BCs (13) are satisfied. For unstable eigenvalues, the solution-averaged Doppler-Coriolis shift should vanish according to Eq. (5), so that

$$
\sigma=\bar{V}[\chi(r ; \omega)] \equiv \frac{\int \rho\left[r \Omega_{0}|\boldsymbol{\xi}|^{2}+\mathrm{i} \bar{v}_{\theta}\left(\chi^{*} \xi_{\theta}-\chi \xi_{\theta}^{*}\right)\right] d r}{\int \rho|\boldsymbol{\xi}|^{2} r d r} \quad \text { (solution path) }
$$

Here, the term with $\Omega_{0}$ represents the Doppler part and the term with $\bar{v}_{\theta} \equiv v_{\theta} / r$ represents the Coriolis part of the shift. To evaluate the volume integrals, one would need to substitute the expressions for $\xi_{\theta}$ and $\xi_{z}$ [obtained from Eqs. (A6) and (A7) of Appendix A] in terms of the solutions $\chi$ and $\Pi$ of the ODE (20).

In the Spectral Web method, instead, the iteration on the eigenvalues is performed by determining the zeros of the two components of the complementary energy according to the scheme (7). The explicit expressions for $W_{\text {com }}$ are given by Eqs. (18), (40) and (42) of Paper I for the left, right and mixed internal solutions, respectively. For the left solutions of a finite length cylinder, the complementary energy reduces to

$$
W_{\mathrm{com}}^{\ell}[\chi(r ; \omega)]=\pi L\left(\chi^{*} \Pi\right)_{r=a} \Rightarrow\left\{\begin{array}{ll}
\pi L\left(\chi_{1} \Pi_{2}-\chi_{2} \Pi_{1}\right)_{r=a}=0 & \text { (solution path) } \\
\pi L\left(\chi_{1} \Pi_{1}+\chi_{2} \Pi_{2}\right)_{r=a}=0 & \text { (conjugate path) }
\end{array} .\right.
$$

Similar simple expressions are easily derived for the other solutions. Whereas the relation (22) for the solution path is physically quite significant, the equivalent relation (23)(a) 
is clearly to be preferred since it just requires evaluation of an expression involving the known boundary data of $\chi$ and $\Pi$.

As pointed out in Paper I, the numerical implementation of the Spectral Web method involves two stages, which may be executed independently, viz.

(a) Contour plotting of the full Spectral Web by solving Eqs. (23) in the strip of the complex $\omega$-plane where the unstable eigenvalues are restricted to lie according to Eq. (22);

(b) Separate eigenvalue search by, first, constructing the solution path by solving Eq. (23)(a) and, next, finding the eigenvalue(s) and associated eigenfunctions on that path by determining the zeros of the alternator.

In this manner, a physically meaningful structure is obtained that connects all eigenvalues in the complex $\omega$-plane. The significant implications for the spectral theory of stationary equilibria will be demonstrated by the explicit examples of the following sections.

The ideal MHD spectrum of a static cylindrical plasma obeys a fundamental oscillation theorem: ${ }^{10}$ the eigenfrequencies are monotonic in the number of nodes of the eigenfunction for values of $\omega$ that lie outside the ranges of the continua $\left\{ \pm \omega_{\mathrm{A}}\right\}$ and $\left\{ \pm \omega_{\mathrm{S}}\right\}$ and the apparent singularities $\left\{ \pm \omega_{\mathrm{s} 0}\right\}$ and $\left\{ \pm \omega_{\mathrm{f} 0}\right\}$. This property appears restricted to static equilibria because the eigenvalue only enters as the real parameter $\omega^{2}$ so that the eigenfunctions are essentially real. Because $\widetilde{\omega}(x)$ is real for $\nu=0$, this remains true for the stable oscillations of stationary equilibria so that the real part of the spectrum is still monotonic outside the ranges $\left\{\Omega_{\mathrm{A}}^{ \pm}\right\},\left\{\Omega_{\mathrm{S}}^{ \pm}\right\},\left\{\Omega_{\mathrm{s} 0}^{ \pm}\right\},\left\{\Omega_{\mathrm{f} 0}^{ \pm}\right\}$, and $\left\{\Omega_{0}\right\}$, as proved in Ref. 11 . These ranges frequently leave very little space for monotonic real discrete modes and, most important, the theorem does not apply to the complex unstable part of the spectrum.

Fortunately, a generalization of the oscillation theorem to stationary equilibria could be constructed by exploiting a quantity that was called the alternator. ${ }^{1,5}$ This involves the quotient of the boundary values of $\chi$ and $\Pi$ so that it is directly related to the complementary energy. For the left, right, and mixed solutions this yields, respectively:

$$
\begin{array}{rlrl}
W_{\mathrm{com}}^{\ell} & =\pi L\left(\chi^{*} \Pi\right)_{r_{\mathrm{r}}}=\pi L|\Pi|_{r_{\mathrm{r}}}^{2} R^{\ell *}, & & R^{\ell} \equiv \chi\left(r_{\mathrm{r}}\right) / \Pi\left(r_{\mathrm{r}}\right), \\
W_{\mathrm{com}}^{\mathrm{r}}=\pi L\left(\chi^{*} \Pi\right)_{r_{\ell}}=\pi L|\Pi|_{r_{\ell}}^{2} R^{\mathrm{r} *}, & & R^{\mathrm{r}} \equiv \chi\left(r_{\ell}\right) / \Pi\left(r_{\ell}\right), \\
W_{\mathrm{com}}^{\mathrm{mix}} & =-\pi L\left(\chi^{*} \llbracket \Pi \rrbracket\right)_{r_{\text {mix }}}=-\pi L|\chi|_{r_{\text {mix }}}^{2} R^{\mathrm{mix}}, & & R^{\mathrm{mix}} \equiv \llbracket \Pi\left(r_{\text {mix }}\right) \rrbracket / \chi\left(r_{\text {mix }}\right) .
\end{array}
$$

where the various endpoints of the integration are indicated by $r_{\mathrm{r}}, r_{\ell}$ and $r_{\operatorname{mix}}$, and the superscripts $\ell, \mathrm{r}$ and mix on $\chi$ and $\Pi$ are omitted for simplicity. Along the solution path, where $W_{\text {com }}$ is real, this alternator is also real, it vanishes at the eigenvalues, and it is monotonic in between the zeros of the boundary values of the other variable (where the alternator blows up). Similarly, along the conjugate path, where $W_{\text {com }}$ is purely imaginary, the alternator vanishes at the eigenvalues, and its imaginary part is monotonic in between the zeros of the boundary values of the other variable. The behavior of the alternator as a tangent facilitates extremely rapid convergence to the eigenvalues. The monotonicity properties of the alternators were proved in Appendix A of Paper I.

The mixed representation is by far the most flexible one since it involves the free parameter $r_{\text {mix }}$, where the left and right solutions are joined. Integrating in a fixed direction starting from one boundary frequently runs into the problem that the solution becomes very imprecise at the opposite boundary, e.g. due to very low densities or other near-singularities. This problem is usually solved by a judicious choice of the value of $r_{\text {mix }}$. Another use of this parameter is the possibility to transform a solution path with gaps into one that is locally 
connected and, thus, establishing the order of the sequences of eigenvalues across those gaps. Recall from Paper I that the proof of definiteness of the sequencing integral exploits an equality [Eq. (A19) of Paper I] in terms of a quadratic form $X$, which would fail if that quantity vanishes somewhere along the solution path. That is precisely where the solution path would become disconnected, as we will see in Sec. IV.

Most examples below exploit the mixed representation with the general form of the spectral differential equations (20) for the real and imaginary components of $\chi$ and $\Pi$, where the sole simplification will be the specification of the equilibrium variables according to Eq. (8). The solutions are obtained by 'shooting' from the left [satisfying the left BC, $\chi(0)=0$ ] and from the right [satisfying the right $\mathrm{BC}, \chi(1)=1$ ], and joining the two fragments in some internal point $x=x_{\text {mix }}$ by equating $\chi$ there. According to Eq. (24)(c), the jump $\llbracket \Pi \rrbracket$ determines both the complementary energy $W_{\text {com }}$ for the determination of the Spectral Web and the alternator for the accurate eigenvalue search. This procedure is incorporated in a computer program, called ROC (= rotating cylinder).

The Spectral Web method is here applied to a selection of some of the classical MHD instabilities, viz. to the internal kink modes of a force-free magnetic field subjected to linear shear flow (Section III), and to the Rayleigh-Taylor instability of a rotating theta-pinch (Section IV) and the Magneto-Rotational Instability (MRI) of an accretion disk (Section V). The spectra of the shear flow equilibria are governed by the Doppler shift profile $\Omega_{0}(r)$, the rotating equilibria attain in addition the complicating Coriolis effects. The main thrust is to demonstrate how this new method facilitates the investigation of all flow-driven instabilities by means of the very intriguing geometries of the Spectral Web, which puts an order into the multitude of complex eigenvalues that was absent heretofore.

\section{INTERNAL KINK MODES IN FORCE-FREE MAGNETIC FIELDS}

Our first example is about the effects of a linear flow field on the classical, current driven, internal kink instability. Consider a cylindrical equilibrium with a force-free magnetic field of constant $\alpha$, and parabolic density and velocity profiles:

$$
B_{z}(r)=J_{0}(\alpha r), \quad B_{\theta}=J_{1}(\alpha r), \quad \rho(r)=\rho_{0}\left(1-r^{2}\right), \quad p(r)=p_{0}, \quad v_{z}=v_{0}\left(1-r^{2}\right) .
$$

Here, $J_{0}$ and $J_{1}$ are the Bessel functions of zeroth and first order, and the constant pressure $p_{0}$ is taken to be large enough to assume incompressibility $\left(\gamma p_{0} \gg 1\right)$ for the modes. We will show that the velocity field changes the spectral properties of this equilibrium completely, in particular the parameter domains and the character of the instabilities of a force-free magnetic field. This is relevant for many astrophysical plasmas. One example is the filamentary structure of the penumbra (the outer region) of sunspots where large flows are observed, ${ }^{12}$ with possible implications for reconnection, heating, flares and coronal mass ejections.

But, first, recall some relevant results on the static case. In the context of observed regular magnetic field structures in the Crab Nebula, Woltjer ${ }^{13}$ studied the stability of force-free fields of constant $\alpha$ and concluded them to be stable against axi-symmetric perturbations. In contrast to the widely held belief at that time this to be true in general, Voslamber and Callebaut $^{14}$ then showed that a cylindrical force-free magnetic field of constant $\alpha$ is actually unstable to internal $|m|=1$ kink modes for $\alpha a>3.176$. Their analysis crucially exploited the concept of independent sub-intervals, introduced in the seminal paper ${ }^{2}$ by Newcomb, to account for the enormously stabilizing influence of rational surfaces where the current density perturbation becomes infinite, similar to the influence of a perfectly conducting wall at 
those positions (see the left panes of Fig. 2 below). Next, Goedbloed and Hagebeuk ${ }^{15}$ computed the eigenfunctions and growth rates of the instabilities and showed them to smoothly join the different independent sub-intervals, with rapid changes across the rational surfaces characteristic for internal kink modes (see the right panes of Fig. 2). The growth rates turned out to be considerable. Also recall that force-free magnetic fields of constant $\alpha$ play an important role in Taylor's theory of relaxation and magnetic reconnection, ${ }^{16}$ as applied to reversed field pinches,${ }^{17}$ spheromaks, ${ }^{18}$ and magnetic structures in the solar corona. ${ }^{19}$

\section{A. Intricacies: Stability of static force-free fields}

The stability of a cylindrical force-free magnetic field is extremely complex, already for the static case, but it touches upon the basics of MHD spectral theory. Hence, we here subject the topic with extra scrutiny. In Fig. 1, we have reconstructed the VoslamberCallebaut stability diagram, ${ }^{14}$ in terms of the relevant dimensionless parameters $k / \alpha$ and $\alpha a$, but extended with the higher radial modes needed for the present analysis. In the limit of marginal stability $(\omega=0)$ for incompressible modes $(\gamma p \rightarrow \infty)$ of static equilibria, the spectral equation (12) has the explicit solution $\chi=r Q_{r} / F$ in terms of the radial magnetic field perturbation $Q_{r}$ and the parallel gradient operator $F=m B_{\theta} / r+k B_{z}$, where

$$
\begin{aligned}
r Q_{r} & =C\left[m(\alpha-k) J_{m}\left(\sqrt{\alpha^{2}-k^{2}} r\right)+k r \sqrt{\alpha^{2}-k^{2}} J_{m-1}\left(\sqrt{\alpha^{2}-k^{2}} r\right)\right], \\
F & =(1 / r)\left[m J_{1}(\alpha r)+k r J_{0}(\alpha r)\right] .
\end{aligned}
$$

Naively, one expects that subjecting the above (left) Bessel function solution for $Q_{r}(r)$ also to the pertinent right $\mathrm{BC}, Q_{r}(a)=0$, would yield the marginal solutions of the problem. The corresponding parameter relations yield the dashed black curves of Fig. 1. From monotonicity, one would then expect the $n=1,2, \ldots$ eigenvalues to lie in the areas to the right of the respective curves. Those areas are indicated by $n=1, n=2$, etc., and shaded increasingly darker black. That conclusion would be fundamentally wrong though since it ignores the ideal MHD constraint that the solution $\boldsymbol{\xi}$ should be square integrable, i.e. have

finite energy content. That condition involves the function $F$ relating $\chi$ and $r Q_{r}$. Since that relation does not hold in resistive MHD, the indicated areas are precisely the parameter regions where the $n=1, n=2$, etc. tearing modes are unstable. Those modes exponentiate with a broken power of the resistivity, so that they are negligible on the ideal MHD time scales of interest in the present context.

Accounting for the ideal MHD constraint involves the additional consideration of the effects of the singularities of $\chi$, i.e. of the zeros of $F(r)$. They provide the sequence of, what Newcomb ${ }^{2}$ has termed, independent sub-intervals, labeled $n=1,2, \ldots$, which is crucial for the stability analysis of the ideal MHD modes. For the intervals $n \geq 2$, the solution (26) for $r Q_{r}$ should then be replaced by the expression which vanishes at the left boundary of that interval, i.e. it should be extended with the appropriate Bessel functions of the second kind. Rather than exploiting those explicit expressions, it is more convenient to just integrate the ODEs for $Q_{r}$ and $F$, imposing the modified BCs on $Q_{r}$ but, of course, still imposing $F(0)=0$ on $F$. At this point it is expedient to note that the problem can be reduced to a one-parameter family, in terms of $\bar{k} \equiv k / \alpha$ alone (ignoring the dependence on $m$ since only 
the $|m|=1$ modes are unstable), by exploiting the scaled radial variable $\bar{r} \equiv \alpha r$ :

$$
\begin{aligned}
& \frac{d}{d \bar{r}}\left[\frac{\bar{r}}{m^{2}+\bar{k}^{2} \bar{r}^{2}} \frac{d}{d \bar{r}}\left(\bar{r} Q_{r}\right)\right]+\left[\frac{\bar{r}^{2}}{m^{2}+\bar{k}^{2} \bar{r}^{2}}\left(1+\frac{2 m \bar{k}}{m^{2}+\bar{k}^{2} \bar{r}^{2}}\right)-1\right] Q_{r}=0 \\
& \frac{d}{d \bar{r}}\left(\frac{\bar{r}^{3}}{\widehat{\widehat{D}}} \frac{d F}{d \bar{r}}\right)+\frac{\bar{r}^{3}}{\widehat{D}}\left(1+\frac{2 m \bar{k}}{\widehat{D}}\right) F=0, \quad \widehat{D}(\bar{r}) \equiv m^{2}+\bar{k}^{2} \bar{r}^{2}+2 m \bar{k}
\end{aligned}
$$

Indicating the zeros of $F$ by $\bar{r}_{n}$, the parameter relations $F\left(\bar{r}_{n}=\alpha a\right)=0$ yield the red curves of Fig. 1. Note the remarkable feature of the ODE for $F$ to develop an apparent singularity $\widehat{D}(\bar{r})=0$ in the radial interval for values of $\bar{k}$ below $-\frac{1}{2} m$ (indicated by the red dashes). This marks the emergence from the origin of an additional zero in the solution $F$, so that the value of $n$ increases by 1 there. The drawn black curves in Fig. 1 indicate the ideal MHD parameter relations $Q_{r}\left(\bar{r}_{n}=\alpha a\right)=0$ where the modified solutions $Q_{r}$ satisfy both BCs of the pertinent sub-interval. Those curves just intersect (or touch) the red curves at the red dots. For reference, we list the coordinates of the red dots for the first two values of $n$ :

$$
(\alpha a, k / \alpha)=\left\{\begin{array}{lll}
(3.1750,0.2725),(3.8317,0.0),(4.7444,-0.2365) & (n=1) \\
(6.4828,0.0951),(7.0158,0.0),(7.6202,-0.0865) & (n=2)
\end{array} .\right.
$$

The values for $n=1$ agree with those given in Ref. 14, as they should. For values of $\bar{k} \equiv k / \alpha$ in between those points, the modified solution $Q_{r}$ oscillates just a little bit faster than $F$ so that that solution has at least one zero in the open sub-interval, indicating ideal MHD instability for that sub-interval. Since, for given $n$, all those intervals are identical in terms of the rescaled variable $\bar{r}$ (but shrinking in size in terms of the physical variable $r$ ), this oscillatory behavior does not change with larger values of $\alpha a$. Hence, the ideal MHD internal kink instability regions lie to the right of the black curves, in between the intersections with the red curves, i.e. in the red shaded strips which become ever narrower and are increasingly darker shaded for larger $n$. Note the very loose connection between the ideal (red) and the resistive (black) instability regions!

Some representative parameter choices for the equilibria and associated perturbations are indicated by the letters $\mathrm{A}-\mathrm{E}$ in Fig. 1. For the static case, the solutions of a marginal mode and corresponding eigenfunction are shown in Figs. 2(a) for the internal kink instability in the $n=1$ strip (case A), and in Figs. 2(b) and 2(c) for the two internal kink instabilities in the $n=2$ strip within the $n=1$ strip (case $\mathrm{C}$ ). The latter two are distinguished by being mainly localized inside or outside the first singularity. In the following, these solutions will be subjected to a flow field and shown to become much more unstable. This is demonstrated by moving into parameter regimes that are resistively unstable, but stable in ideal MHD for static equilibria. The cases $\mathrm{B}(n=1$ stable in the static case), $\mathrm{D}$ ( $n=1$ unstable but $n=2$ stable in the static case), and $\mathrm{E}$ (both $n=1$ and $n=2$ stable in the static case) will be shown to become unstable for the two kinds of modes, with quite different eigenfunctions and much larger growth rates.

\section{B. Internal kink modes with flow}

We will now demonstrate how a flow field completely changes the stability properties of this configuration and how the Spectral Web facilitates the search of the complex eigenvalues. Figure 3 shows the rather elementary Spectral Web for the $n=1$ internal kink instability 
for the configuration of case A, subjected to a parabolic flow profile with associated Doppler frequencies $\Omega_{0}=0.8\left(1-x^{2}\right)$, where $x \equiv r / a$. The corresponding eigenfunction is shown in Fig. 4(a). As is evident from Fig. 3, the Spectral Web is an excellent guide to find the eigenvalues. Contour plotting may be restricted to the strip $\Omega_{0, \min } \leq \sigma \leq \Omega_{0, \max }$ where the solution path should be located according to Eq. (5). This corresponds with the range $\left\{\Omega_{0}(r)\right\}$ of Doppler shifts on the radial interval $0 \leq r \leq a$ depicted by the dashed line in the upper part of Fig. 3. The incompressible ideal MHD continuum frequencies $\left\{\Omega_{\mathrm{A}}^{+}(r)\right\}$ and $\left\{\Omega_{\mathrm{A}}^{-}(r)\right\}$, depicted in red, intersect this range at the Doppler frequency $\Omega_{0} \approx 0.437$ of the rational surface at $r / a \approx 0.674$. For the block shape (in $\xi_{r}$ ) of a marginal internal kink mode, having an infinite contribution from the jump at the rational surface, the real part of the eigenfrequency would precisely have that value. However, as shown in Fig. 4(a), the eigenfunction is much more rounded than that so that the actual real part $\sigma \approx 0.478$ of the eigenfrequency of the mode is larger, due to the contribution of the eigenfunction at smaller values of $r$ (where $\Omega_{0}$ is larger). This also explains the shape of the solution path of Fig. 3 . Intuitive explanation of the disconnected curves of the conjugate path is less clear. Probably, the extremum of the underlying continuum $\left\{\Omega_{\mathrm{A}}^{-}\right\}$is responsible for that. Since there are no eigenvalues located on the right branch of the conjugate path, this is not important here. (A different situation occurs in subsection IIIC below.)

Comparing Fig. 4(a) with Fig 2(a) for the internal kink mode inside the $n=1$ instability domain of the static equilibrium (case A of Fig. 1), the growth rate is about a factor 2.0 larger than without flow. Fig. 4(b) shows an internal kink mode far outside the $n=1$ instability domain of the static equilibrium (case B of Fig. 1): the near marginal mode has a clear internal kink mode signature. Evidently, for plasmas with flow, the current driven instabilities of a force free magnetic field have much larger growth rates and much wider instability domains than for static equilibria.

Figure 5 shows the Spectral Web for the $n=1$ and $n=2$ internal kink instabilities of the configuration of case $\mathrm{D}$, subjected to a parabolic flow profile with associated Doppler frequencies $\Omega_{0}=1.152\left(1-x^{2}\right)$. This case was used in Figures 1 and 2 of Paper 1 for the purpose of illustrating the different concepts used in the new method, where the first figure showed the trivial case of a constant velocity and the second one demonstrated the complete change of the Spectral Web, with the appearance of two modes instead of one, for a genuine flow profile. For the present Fig. 5, the magnitude of that flow has been reduced to bring out the new class of instabilities that will be discussed in the next subsection. The first thing that strikes is that the solution path is no longer a single curve, as one might have expected from the fact that it is actually the counterpart of the imaginary axis (the locus of instabilities for static equilibria), but split in two. The rather straight right sub-path contains the $n=1$ internal link instability (labeled $\mathrm{I}_{1}$ ) and the loop contains the $n=2$ internal link instability (labeled $\mathrm{I}_{2}$ ). Actually, loops of the solution path or the conjugate path are a bonus in this method since they necessarily contain an eigenvalue due to the monotonicity of the alternator along these paths. Also noteworthy is the useful visual difference between the conjugate paths of Fig. 2 of Paper 1 and Fig. 5 of the present paper, where the latter right away leads to the new class of modes (labeled $G_{i}$ ) which is absent in the former.

The eigenfunctions of the two internal kink modes $I_{1}$ and $I_{2}$ of Fig. 5 are shown in Fig. 6 . The growth rate of the $n=1$ eigenmode, shown in Fig. 6(a), is slightly smaller (a factor 0.93 ) than in the corresponding static case (not shown but similar to Fig. 2(b)), but the amplitude of the eigenfunction in the second "independent" sub-interval is effectively suppressed. On the other hand, the $n=2$ mode is now unstable and even has a larger growth rate than the 
$n=1$ mode, whereas the eigenfunction, shown in Fig. 6(b), has completely lost its signature of being localized to the second "independent" sub-interval. Also notice the peaks in the eigenfunctions at the locations of the underlying continuous spectra, where $\sigma_{\mathrm{EF}}=\Omega_{\mathrm{A}}^{ \pm}(r)$. Although these continuum frequencies are far away from the complex eigenfrequencies of the instabilities, their influence on the discrete eigenfunctions is quite noticeable. Finally, for fixed $\alpha a=8.0$ increasing the value of $k / \alpha$ to $k / \alpha \approx 3.0$ (case $\mathrm{E}$ in the stability diagram of Fig. 1, just above the stability boundary for the static $n=1$ modes but far above that for the static $n=2$ modes), both modes have become stable nearly simultaneously: the stability domains of the internal kink modes are extended far into the resistive regions. Hence, the Spectral Web method reveals how flow completely changes the spectrum of MHD instabilities, but also how it brings about new modes, as we will see next.

\section{A truly Global Alfvén Instability}

It remains to discuss the sequence of eigenvalues labeled $\mathrm{G}_{i}$, emerging from the continua (the grey horizontal axis of Fig. 5) and highlighted by the inset of Fig. 5. The inset is a blow up of a part of the $\mathrm{G}_{1}$ loop overarching all other loops of these modes. Two of the corresponding eigenfunctions are depicted in Figs. 7(c) and (d). The real part of the frequency of these instabilities is clearly related to the local maximum $\Omega_{\mathrm{A} \text {,max }}^{+} \approx 0.657$ of the forward Alfvén continuum shown in the radial plots of the continua in the upper part of Fig. 5. Note the logarithmic scale in $\nu$ and the very narrow range in $\sigma$ of the inset, which are needed to exhibit the vary rapid decrease of the growth rates and the clustering towards $\Omega_{\mathrm{A}, \max }^{+}$for increasing index $i$ of the eigenvalues. These instabilities are someway related to the infinite sequence of stable Global Alfvén Eigenmodes (GAEs), ${ }^{20}$ that would be found for real frequencies approaching $\Omega_{\mathrm{A}, \max }^{+}$if that potential cluster point would not be overlapped by the rest of the continua. However, it is evident from the plots in the upper part of Fig. 5 that multiple overlapping occurs so that discrete GAEs cannot exist in this case, they are simply 'swallowed' by the continua.

Although the stable GAEs cannot occur in the present equilibrium, it is useful to digress on them to clarify the relation with the instabilities we are interested in. Actually, the designation 'global' in Global Alfvén Eigenmodes is a misnomer since these modes occur when a local criterion is satisfied, associated with infinitely rapidly oscillating solutions as the cluster frequency is approached. This is quite analogous to the occurrence of interchanges when Suydam's criterion is violated; see the expression (C2) of Appendix C for the modified Suydam criterion for plasmas with flow. [Incidentally, satisfaction of that criterion is not obvious for force free equilibria $\left(p^{\prime}=0\right)$ with flow, because of the large negative term in criterion (C2). We checked though that the criterion for incompressible modes is satisfied at the rational surfaces for the parameters chosen, so that interchanges do not occur in the present case.] Appendix C also presents the modification by flow of the cluster criteria for Alfvén and slow modes. ${ }^{21}$ Those criteria are not valid for the present, incompressible, modes because some of the terms blows up. The two regular singularities $\Omega_{\mathrm{A}}^{+}$and $\Omega_{\mathrm{S}}^{+}$and the apparent singularity $\Omega_{\mathrm{s} 0}^{+}$in between then coalesce into one irregular singularity. ${ }^{22}$ Expansion about that singularity yields the following leading order ODE with solutions:

$$
\frac{d}{d s}\left(s^{2} \frac{d \chi}{d s}\right)+(q s)^{-1} \chi=0, \quad q \equiv \frac{r \sqrt{\rho} \Omega_{\mathrm{A}}^{+^{\prime \prime}}}{2 k B_{\theta}} \Rightarrow \chi=C_{1} \sin (q s)^{-1}+C_{2} \cos (q s)^{-1} .
$$

There is no criterion to be satisfied in this case, so that incompressible stable GAEs always 
occur if one of the Alfvén continua has an extremum, provided that the radial interval may be chosen small enough to exclude overlap with the continuum frequencies of the complete plasma. This local behavior is demonstrated in Figs.7(a) and (b), where the real eigenfunctions of two of the GAEs are plotted on the sub-interval $0.8 \leq r \leq 1.0$. This interval refers to the equilibrium of an annular plasma enclosed by two walls, which is not the equilibrium though we are interested in.

The global eigenfunctions of the instabilities indicated by the labels $\mathrm{G}_{i}$, shown in Figs. 7(c) and (d) for $\mathrm{G}_{1}$ and $\mathrm{G}_{3}$, do exhibit the oscillatory properties of the first and third 'local' GAE, illustrated in Figs. 7(a) and (b), on the outermost part of the plasma, but, equally important, they also exhibit sharp peaks at the three radial locations $r_{1}>r_{2}>r_{3}$ of the embedding continua, where $\Omega_{\mathrm{A}}^{-}\left(r_{1}\right)=\Omega_{\mathrm{A}}^{+}\left(r_{2}\right)=\Omega_{\mathrm{A}}^{-}\left(r_{3}\right)=\Omega_{\mathrm{A}, \max }^{+}$. The large contribution of these peaks to the eigenfunction is crucial to produce an instability with the eigenvalue on the solution path, i.e. having a real part equal to the solution-averaged Doppler shift, $\sigma=\Omega_{0, \text { av }} \approx \Omega_{\mathrm{A} \text {,max }}$, according to Eq. (5). Note that the local GAE cannot possibly be unstable by itself since it has $\Omega_{0}\left(r_{\max }\right) \ll \Omega_{\mathrm{A} \text {,max }}^{+}$, much too small to be on the complex branch of the solution path. But at the three mentioned continuum locations we have $\Omega_{0}\left(r_{1}\right)<\Omega_{\mathrm{A}, \max }^{+}<\Omega_{0}\left(r_{2}\right)<\Omega_{0}\left(r_{3}\right)$, so that those three contributions on average produce the proper global localization of the eigenfunction needed for the eigenfrequency to be on the solution path.

For the same reason, the continuum profiles shown in Fig. 3 for the first instability domain of the force-free field equilibrium at once explain why GAIs do not occur in that configuration. Those profiles also do have an extremum $\Omega_{\mathrm{A} \text {,max }}^{-}$, with an associated GAE for a narrow annular plasma, but, in that case, the location $r_{1}$ of the overlapping continuum frequency with $\Omega_{\mathrm{A}}^{+}\left(r_{1}\right)=\Omega_{\mathrm{A}, \max }^{-}$is on the wrong side of the profile $\Omega_{0}(r)$ so that it cannot produce the localization needed to pull the solution over onto the solution path.

In conclusion, in contrast to the stable GAEs, the present instabilities are truly global modes, which we will call Global Alfvén Instabilities (GAIs). One could say that they are GAEs driven unstable by the delocalization caused by the proximity of the continua, requiring both plasma current and plasma flow. In those respects they are similar to Toroidal Flow-induced Alfvén Eigenmodes (TFAEs) ${ }^{23}$ and Toroidal Alfvén Eigenmodes (TAEs) that are driven unstable by the interaction with the flow of fusion-born fast particles; see, e.g., Ref. 24 and references therein. Finally, concerning the Spectral Web method, it is of interest to note that the GAIs were discovered because the tiny loop of the conjugate path at $\sigma \approx 0.68$ in Fig. 2 of Paper I suggested unknown modes there. Without the guidance of the conjugate path it is unlikely that they would have been discovered by another method.

\section{RAYLEIGH-TAYLOR INSTABILITIES IN ROTATING THETA-PINCHES}

Our second example concerns the $|m|=1$ modes of a class of rigidly rotating equilibria of theta pinch-like plasma configurations in the straight cylinder approximation, as exemplified by the ordinary theta pinch $^{25,26}$ and the Field Reversed Configuration (FRC). ${ }^{27,28}$ Although of historical interest only, we will treat the rather disastrous instabilities of the rotating theta pinch more extensively since they provide an excellent illustration of the Spectral Web method which also applies to the more subtle instabilities of modern devices.

The stability of the rotating theta pinch is of fundamental interest since it was used in the original literature on the stabilization of MHD instabilities by the finiteness of the ion gyro radius, ${ }^{29,30}$ which is least effective for the $|m|=1$ modes. (Hence, the interest in these modes at the time, though the $|m|>1$ modes grow faster yet.) This literature contains 
some confusing results that have not been clarified before. In particular, Taylor ${ }^{30}$ makes the erroneous remark that the growth rate of the instability for vanishing longitudinal mode number $(k=0)$, i.e. the imaginary part of his expression for the complex frequency,

$$
\omega=\Omega[m-\operatorname{sgn}(m) \pm \mathrm{i} \sqrt{|m|-1}]
$$

is "quite independent of the plasma profile". According to this expression, the Coriolis shift $(\Delta \sigma)_{\mathrm{C}}=-\operatorname{sgn}(m) \Omega$ then precisely cancels the Doppler shift $(\Delta \sigma)_{\mathrm{D}}=m \Omega$ so that the real part of the complex frequency also vanishes. This would imply marginal stability of the $|m|=1$ modes for these configurations in general. However, contrary to Taylor's remark, this expression is only valid for the special case he investigated, viz. a theta pinch with a step function density profile without a wall. Effectively, for the incompressible $|m|=1, k=0$ modes of that equilibrium, there is no perturbation at all since that 'perturbation' of the plasma column simply corresponds to a translation to a new equilibrium state with the same properties. A detailed numerical calculation by Freidberg and Wesson ${ }^{25}$ appeared to confirm Taylor's result, by searching in the complex $\omega$-plane for solutions in the neighborhood of the imaginary axis as suggested by Eq. (30). A later study by Freidberg and Pearlstein ${ }^{26}$ of the finite ion gyro radius effects on these modes actually found substantial deviations from Eq. (30) for an additional class of internal $|m|=1$ modes. The Spectral Web method will highlight one of the intricacies involved of the stability of rotating stationary equilibria, viz. that the more local internal $|m|=1$ modes actually grow faster than the most global one!

Diffuse rigidly rotating theta pinch equilibria are described by satisfying Eq. (8) with constant angular frequency $\Omega \equiv v_{\theta} / r$ and the following choice of the profiles:

$$
\begin{aligned}
\rho(r) & =\rho_{0} \operatorname{sech}^{2} f(x), \quad p(r)=p_{0} \operatorname{sech}^{2} f(x), \\
B_{z}(r) & =B_{\infty}[\delta+(1-\delta) \tanh f(x)], \quad f(x) \equiv \alpha^{2}\left(x^{2}-x_{0}^{2}\right),
\end{aligned}
$$

where $x \equiv r / a$, whereas $p_{0}=\frac{1}{2}(1-\delta)^{2} B_{\infty}^{2}$ and $\Omega=\alpha \sqrt{2 \delta(1-\delta)} B_{\infty} /\left(a \sqrt{\rho_{0}}\right)$. The plasma radius $a$, the maximum density $\rho_{0}$, and the asymptotic value $B_{\infty}$ of the magnetic field merely provide parameters to make the basic quantities dimensionless, like the unit plasma interval $0 \leq x \equiv r / a \leq 1$, the longitudinal wave number $\bar{k} \equiv k a$, and the complex frequency $\bar{\omega} \equiv \omega a \sqrt{\rho_{0}} / B_{\infty}$. Hence, only three genuinely free dimensionless parameters remain, viz. the normalized radius $x_{0}$ at maximum density, the stretching parameter $\alpha$, and the magnetic field deviation parameter $\delta$. The parameter $x_{0}$ is the distinguishing one for the FRC, but it vanishes for the theta pinch equilibrium. The parameters $\alpha$ and $\delta$ are more conveniently represented by the dimensionless pressure, $\beta \equiv 2 \bar{p}_{0}=(1-\delta)^{2}$, and the dimensionless angular rotation frequency, $\bar{\Omega}=\alpha \sqrt{2 \delta(1-\delta)}$. We will exploit the latter parameters, dropping the bars. A representative equilibrium is depicted in Fig. 8.

The rotating theta pinch equilibrium of Fig. 8 has decreasing density and pressure profiles over the whole plasma domain, and a uni-directional increasing magnetic field profile. Since the stabilizing magnetic field terms $k^{2} B_{z}^{2}$ vanish for the $k=0$ modes, the instabilities are essentially hydrodynamic, Rayleigh-Taylor, instabilities driven by the negative density gradient and the centrifugal acceleration. ${ }^{31}$ Magnetic stabilization occurs for the $k \neq 0$ modes, but a large range $0 \leq|k| \leq\left|k_{0}\right|$ remains unstable. Similar conclusions hold for the straight cylinder approximation of the FRC equilibrium, but the instability is less severe because the density increases in the region $0 \leq x \leq x_{0}$, so that the inner region is stable, whereas the magnetic field reversal, and associated singularity $\mathbf{k} \cdot \mathbf{B}=0$, close to $x=x_{0}$ significantly modifies the modes at marginal stability. 


\section{A. Hydrodynamic modes $(k=0)$}

The Spectral Web for the compressible $m=1, k=0$ modes of the rotating theta pinch equilibrium is shown in Fig. 9 (note Ref. 32). One immediately notices the interlacing structure of the solution path (in red) and the conjugate path (in blue), with genuine and false eigenvalues (EVs) alternating at the intersections. Furthermore, the solution path is not a single curve, as one might have expected, but it is split into a separate open curve with the most global $n=1$ mode on it and a closed loop with the $n=2$ mode and all the rest of infinitely many clustering modes. The latter have a Doppler shifted frequency $\widetilde{\sigma} \equiv \sigma-m \Omega$ which tends to zero, whereas the most global modes have a large Coriolis shift, opposite to the Doppler shift, such that the combined Doppler-Coriolis shift $\bar{V}$, and hence $\sigma$, becomes very small. The most striking feature is already mentioned, viz. that the $n=2$ mode has a larger growth rate than the $n=1$ mode. Such behavior never occurs for static equilibria. This calls for justification of the labels $n=1, n=2$, etc.

For the $n \geq 2$ modes, the justification of the labeling is provided by the monotonicity of the alternator along the solution path, which yields a well-ordered sequence toward the cluster point $\omega=m \Omega$. This sequence does not include the $n=1 \mathrm{EV}$ s though since they are on a separate sub-path. At this point, it is important to note that the Spectral Webs of Fig. 9 and 11 were obtained for a specific choice of the parameter $x_{\operatorname{mix}}\left(\right.$ viz. $x_{\operatorname{mix}}=0.7$ ) which is optimal for the present problem. Whereas the complex eigenvalues are independent of this choice (as they should be), the geometries of the solution path and of the conjugate path are not. In particular, their split into disconnected sub-paths, as in Fig. 9, depends on the value of $x_{\text {mix }}$. Frequently, disconnected sub-paths may be merged by just changing that value. However, in this case, the attempt of connecting the sub-paths by a different choice of $x_{\text {mix }}$ fails: for $x_{\text {mix }}=0.7493$, the topology of the solution path is still as shown in Fig. 9, but for $x_{\text {mix }}=0.7494$, it has abruptly changed into one where now $n=1$ is on a closed loop and the $n \geq 2$ EVs are on an open curve. Apparently, a possible merger (with $|X|=0$ at the reconnection point) is simply avoided. However, the conjugate path does connect the two EVs and, with the proof of monotonicity of the imaginary part of the alternator along the conjugate path (see Appendix A of Paper I), this provides clinching evidence for the validity of the collective labeling $n=1, n=2$, etc.

To get more grip on the physics of these instabilities, some explicit analysis is expedient. First, consider the local (high $n$ ) modes. For $k=0$, the four MHD continua $\left\{\Omega_{\mathrm{A}}^{ \pm}(r)\right\}$ and $\left\{\Omega_{\mathrm{S}}^{ \pm}(r)\right\}$, defined in Eq. (17), collapse into the hydrodynamic (HD) flow continuum, ${ }^{33}$ $\left\{\Omega_{0}(r)\right\}$, which is infinitely degenerate here since $\Omega_{0}=m \Omega$ is constant. Approaching this continuum tangentially to the vertical line $\widetilde{\sigma} \equiv \sigma-m \Omega=0$ in the $\omega$ plane, the differential equation obtained from Eq. (12) in the pertinent approximation,

$$
\widetilde{\omega}^{2} \frac{d}{d r}\left(\rho r \frac{d \chi}{d r}\right)+m^{2} \Omega^{2}\left(\rho^{\prime}-\frac{r \rho^{2} \Omega^{2}}{\gamma p+B^{2}}\right) \chi=0
$$

gives rise to an infinite sequence of ever more rapidly oscillating solutions. This is associated with a cluster sequence of unstable discrete modes (approaching the red dot of Fig. 9) when the well known local stability criterion for convective, Rayleigh-Taylor, instability,

$$
C_{\text {stab }} \equiv \frac{1}{\alpha^{2}}\left(\frac{\rho^{\prime}}{\rho r}-\frac{\rho \Omega^{2}}{\gamma p+B^{2}}\right)>0,
$$

is violated. This is the case for the equilibrium of Fig. 8 over the whole plasma (as shown by the green curves). The second term, representing additional destabilization due to com- 
pressibility, becomes increasingly more important at higher densities, faster rotations, and smaller magnetic field strengths. For the present equilibrium, the first term dominates over most of the plasma (except for the neighborhood of the magnetic axis), so that the assumption of incompressibility appears to be reasonable for most modes (except for the higher $n$ ones, and the marginal $k \neq 0$ modes discussed below).

To understand the overall geometry of the Spectral Web of Fig. 9, it is useful to temporarily make the approximation of incompressibility for the $k=0$ modes. For both global and local modes, the spectral differential equation (12) then reduces to

$$
r \frac{d}{d r}\left(\rho r \frac{d \chi}{d r}\right)-\left(m^{2} \rho+\lambda r \rho^{\prime}\right) \chi=0, \quad \lambda \equiv-m \Omega(2 \widetilde{\omega}+m \Omega) / \widetilde{\omega}^{2},
$$

where $\lambda$ is a convenient eigenvalue replacing $\omega$. This equation does not have the compressibility term of Eq. (33), but it is no longer restricted to the neighborhood of the cluster point $\widetilde{\omega}=0$. For this special case, as noted by Spies, ${ }^{34}$ the complex eigenvalue problem has degenerated into a Sturm-Liouville problem with real eigenvalues $\lambda$ and real eigenfunctions $\chi$, where the actual complex eigenvalues $\omega$ follow from the definition of $\lambda$. One may construct the following quadratic equation for the eigenvalue $\widetilde{\omega}$ from Eq. (34):

$$
\widetilde{\omega}^{2}+2 Q m \Omega \widetilde{\omega}+Q m^{2} \Omega^{2}=0,
$$

where $Q \equiv \lambda^{-1}$ turns out to be a quotient of two integrals,

$$
Q=-\left(1 / m^{2}\right) \int \rho^{\prime}|\chi|^{2} d r / \int \rho\left[(1 / r)|\chi|^{2}+\left(r / m^{2}\right)\left|\chi^{\prime}\right|^{2}\right] d r
$$

Hence, the eigenvalues of the instabilities are given by

$$
\omega=m \Omega[1-Q \pm \mathrm{i} \sqrt{Q(1-Q)}] .
$$

By eliminating $Q$, one finds that the eigenvalues are located on a circle $\left|\omega-\frac{1}{2} m \Omega\right|=\frac{1}{2}|m| \Omega$ through the origin (where $Q=1$ ) and that the cluster point (where $Q=0$ ) is approached by EVs associated with eigenfunctions that have an increasing number of oscillations. ${ }^{34}$ It is clear that instability occurs for $\rho^{\prime}<0$, when the eigenvalue $Q$ is in the range $0<Q<1$. However, the value of $Q$ and, hence, the values of $\sigma$ and $\nu$ cannot be evaluated a priori: they depend on the non-trivial solution $\chi$ of the boundary value problem (34), (13). Since the effects of compressibility are small for most of the $k=0$ modes of the present equilibrium, the eigenvalues shown in Fig. 9 are rather close to the mentioned circle.

Comparison of the expression (37) with the general expression (4) for the eigenvalues yields the following intriguing relations for the solution averages of the Doppler-Coriolis shift and the potential energy for this case:

$$
\bar{V}=(1-Q) m \Omega, \quad \bar{W}=-(1-Q) m^{2} \Omega^{2} .
$$

They clearly demonstrate that, for the investigation of the stability of stationary equilibria, the two quadratic forms $\bar{W}$ and $\bar{V}$ play equally important roles.

We return to the actual numerical results for the compressible $k=0$ modes. The eigenfunctions for the $n=1$ and $n=2$ modes, shown in Figs. 10(a) and (b), display the usual features of low $n$ modes, where $n=1$ is the most global one. If the plasma column would be more confined (by increasing the value of the stretching parameter $\alpha$ ), the configuration 
would tend to the one discussed by Taylor ${ }^{30}$ and that eigenvalue would approach the origin (where $Q=1$ in the incompressible analysis). Hence, the $n=1$ mode is intrinsically more stable than the $n=2$ mode, which is located farthest away from both the origin and the cluster point (where $Q=0$ ).

The side frame of Fig. 9 shows a zoom of the cluster spectrum, with a further zoom in the bottom frame highlighting the $n=53, \ldots 56$ modes. One notices the characteristic alternation of genuine and false eigenvalues, with monotonicity of the alternator $\llbracket \Pi \rrbracket_{\text {mix }} / \chi_{\text {mix }}$ in between the false ones. This is in perfect agreement with the oscillation theorem for complex eigenvalues permitting counting of modes in the complex $\omega$ plane. The adjacent location of the $n=55$ and 56 modes on the same loop of the conjugate path, which would conflict with this theorem, is only apparent. Small loops of the conjugate path may become entangled with bigger ones, leading to a convoluted structure at very small scales which only becomes visible by further zooming in (as shown for the $n=55$ mode in the bottom side frame of Fig. 9). These higher $n$ compressible modes localize both at the position of maximum $C_{\text {stab }}$ (on the outside) and at the origin (where compressibility is dominant), as shown in Fig. 10(c) for the compressible $n=54$ mode. For comparable growth rate of the incompressible modes, the number of oscillations about the origin decreases, indicative of the next significant difference with static equilibria: for stationary equilibria, compressibility destabilizes.

\section{B. Magnetohydrodynamic modifications $(k \neq 0)$}

The magnetic field completely changes the topology of the Spectral Web and, with it, the location of the unstable eigenvalues, in particular of the more global modes. This is shown in Fig. 11 by the Spectral Web for the $m=1, k=0.1$ modes. For this value of $k$, the two separate branches of the solution path with the $n=1$ and $n=2$ modes on them have merged, but those two modes are still wide apart in the $\omega$-plane, with very different values of $\sigma$. It is convenient to express this real part of the EV in terms of the Coriolis shift, which may be equated with the Doppler shifted frequency $\widetilde{\sigma}$, since, along the solution path,

$$
\sigma=\bar{V}=(\Delta \sigma)_{\mathrm{D}}+(\Delta \sigma)_{\mathrm{C}} \quad \Rightarrow \quad \tilde{\sigma} \equiv \sigma-m \Omega=(\Delta \sigma)_{\mathrm{C}}
$$

As in Fig. 9 for $k=0$, the growth rate $\nu$ of the $n=2$ mode is still much larger than the growth rate for the $n=1$ mode, whereas the Coriolis shift $\widetilde{\sigma}$ is much smaller.

Concerning the higher $n$ modes, the Spectral Web of Fig. 11 illustrates how the majority of the unstable modes of the hydrodynamic cluster spectrum of Fig. 9 is now stabilized by the magnetic field. It also illustrates how the degeneracy of the HD flow continuum is lifted and how the approach of the marginal stability point is now governed by the resulting spread of the MHD continuum frequencies. In particular, the backward continua are well separated from the forward continua, leaving a narrow gap for marginal modes $\omega=\sigma_{\text {marg }}$ to pass:

$$
\begin{array}{rlrl}
\left\{\Omega_{\mathrm{A}}^{-}\right\} & =[-1.6751,1.0375], & & \left\{\Omega_{\mathrm{A}}^{+}\right\}=[1.0706,3.7835], \\
\left\{\Omega_{\mathrm{S}}^{-}\right\}=[0.9781,1.0379], & & \left\{\Omega_{\mathrm{S}}^{+}\right\}=[1.0705,1.1302], \\
\Rightarrow & \left\{\Omega_{\mathrm{S}}^{-}, \Omega_{\mathrm{A}}^{-}\right\}_{\max }<\sigma_{\operatorname{marg}} & <\left\{\Omega_{\mathrm{S}}^{+}, \Omega_{\mathrm{A}}^{+}\right\}_{\min } .
\end{array}
$$

One can clearly see the transition from the pairs of complex modes ( $n=1-4$ are highlighted in the main frame, $n=5-13$ in the side frame) to the pairs of real stable modes $(n \geq 14)$. 
The numbering $n=1,2, \ldots 13$ of the complex eigenvalues (obtained by counting the number of branches of the alternator) smoothly joins that of the stable eigenvalues on the real axis (where $n$ counts the number of nodes of the real eigenfunctions). In order not to clutter the picture, only the two $n=14$ modes are shown since there is a large number of stable modes close to the continua, whereas the highest ones have been 'swallowed' by them.

We finally apply the Spectral Web method to compute the complete curve of the growth rate $\nu(k)$ and of the Coriolis shift $\widetilde{\sigma}(k)$ for all values of $k$, from the most unstable modes at $k=0$ to the marginally stable ones at $k=k_{0}$. Of course, all the underlying Spectral Webs will not be shown. Actually, one of the advantages of this method is that one may restrict the investigation to small pieces of the $\omega$-plane if, in a parameter scan, one already roughly knows where the next eigenvalue will be located. Because of the increasing magnetic field stabilization, the curves shown in Fig. 12 are overall decreasing. At larger $k(k>0.254)$, the dominance of the instability of the $n=2$ modes (indicated by the blue and green curves in Fig. 12) disappears and the $n=1$ modes (indicated by the red and black curves) become more unstable, so that eventually marginal stability is determined by the $n=1$ mode. Note that the values of the growth rate $\nu$ and of the unstable ranges of the wave vector $k$ shown here are completely different from those reported in Ref. 25.

For larger $k$, the values of $\nu$ and $\widetilde{\sigma}$ are also quite different for incompressible and compressible modes (note the deviation of the dashed curves from the drawn ones in Fig. 12). It is clear from the equilibrium profiles shown in Fig. 8 that magnetic field line bending, expressed by the terms $k^{2} B_{z}^{2}$ in the spectral equation, is strongest on the outside so that, eventually, the marginal stability transition at $k=k_{0}$ is determined by the internal region, where compressibility is dominant. This explains the large differences at marginal stability: $k_{0}=0.948$ for the compressible $n=1$ modes $\left(k_{0}=0.548\right.$ for $\left.n=2\right)$, versus $k_{0}=0.792$ for the incompressible $n=1$ modes $\left(k_{0}=0.419\right.$ for $\left.n=2\right)$. Recall that such differences never occur for static equilibria (those numbers would coincide there). Hence, compressibility makes a significant qualitative change.

In conclusion: the Spectral Web analysis of the rotating theta pinch demonstrates that three 'intuitive' assumptions originating from numerous investigations of the stability of static equilibria, viz. (1) that stability is determined by the sign of the potential energy $W$, (2) that the most global modes have the largest growth rate, (3) that it is sufficient for stability to investigate the incompressible modes only, are false for stationary equilibria.

\section{MAGNETO-ROTATIONAL INSTABILITIES IN ACCRETION DISKS}

Our third example concerns the magneto-rotational instability (MRI), independently found by Velikhov ${ }^{35}$ and Chandrasekhar ${ }^{36}$ and later applied by Balbus and Hawley ${ }^{37}$ to clarify the mechanism of accretion onto compact objects. The relevant equilibrium is introduced as case (d) of Sec. II A. We exploit three basic magnitudes referring to the inner edge of the accretion disk, viz. $r_{1}, \rho_{1}$ and $G M_{*}$, to make all variables dimensionless: $\bar{r} \equiv r / r_{1}, \bar{\rho} \equiv \rho / \rho_{1}$, $\bar{v}_{\theta} \equiv\left[r_{1} /\left(G M_{*}\right)\right]^{1 / 2} v_{\theta}, \bar{p} \equiv\left[r_{1} /\left(\rho_{1} G M_{*}\right)\right] p, \bar{B}_{\theta, z} \equiv\left[r_{1} /\left(\rho_{1} G M_{*}\right)\right]^{1 / 2} B_{\theta, z}$, and immediately drop the bars again (which amounts to substituting $r_{1} \equiv 1, \rho_{1} \equiv 1$, and $G M_{*} \equiv 1$ ). A thin disk equilibrium satisfying Eq. (8), with appropriate approximation of the gravitational potential, is then obtained from self-similarity arguments ${ }^{38}$ and an estimate of the viscosity needed for accretion. ${ }^{39}$ On the fast time scale of the pertinent MHD processes, dissipation 
may be neglected and one obtains the following radial equilibrium distributions:

$$
\begin{aligned}
& \rho=r^{-3 / 2}, \quad v_{\theta}=v_{\theta 1} r^{-1 / 2}, \quad p=p_{1} r^{-5 / 2}, \quad B_{\theta}=B_{\theta 1} r^{-5 / 4}, \quad B_{z}=B_{z 1} r^{-5 / 4}, \\
& \text { where } \quad \Omega_{1} \equiv v_{\theta 1}=\left[1-\frac{5}{2} p_{1}-\frac{1}{2} B_{\theta 1}^{2}-\frac{5}{4} B_{z 1}^{2}\right]^{1 / 2} .
\end{aligned}
$$

Instead of the three parameters $p_{1}, B_{\theta 1}, B_{z 1}$, it is convenient to exploit their relative magnitudes and a parameter measuring the radial size of disk:

$$
\epsilon \equiv \sqrt{p_{1}} \ll 1, \quad \beta \equiv 2 p_{1} / B_{1}^{2} \gg 1, \quad \mu_{1} \equiv B_{\theta 1} / B_{z 1} \sim 1, \quad \delta \equiv r_{2} / r_{1} \gg 1 .
$$

This ordering has been chosen such that the angular rotation parameter is of order unity, $\Omega_{1} \approx 1-(5 / 4) \epsilon^{2}$, implying that the rotation is close to Keplerian, whereas the pressure is small enough to warrant the thin disk approximation and the magnetic fields are extremely small, yet important enough to yield the MRIs.

\section{A. Analytical estimates}

To get an estimate of the important mechanisms, we collect some approximate results (see Refs. 8 and 9). Consider axi-symmetric modes, $m=0$, so that the Doppler shift vanishes, $\widetilde{\omega} \equiv \omega$, and the continua transform into the static ones: $\Omega_{\mathrm{A}}^{ \pm}= \pm \omega_{\mathrm{A}}$ and $\Omega_{\mathrm{S}}^{ \pm}= \pm \omega_{\mathrm{S}}$. Moreover, since $\beta$ is so large $(\gamma \beta \gg 1)$, the perturbations are approximately incompressible so that the slow and Alfvén continu virtually coincide. With these approximations, and writing $\omega_{\mathrm{A}} \equiv k B_{z} / \sqrt{\rho}$ and $\mu \equiv B_{\theta} /\left(r B_{z}\right)$, the spectral equation (12) reduces to

$$
r \frac{d}{d r}\left[\rho\left(\omega^{2}-\omega_{\mathrm{A}}^{2}\right) \frac{1}{r} \frac{d \chi}{d r}\right]-k^{2}\left[\rho\left(\omega^{2}-\omega_{\mathrm{A}}^{2}\right)+\Delta-\frac{4\left(k \mu B_{z}^{2}+\rho \Omega \omega\right)^{2}}{\rho\left(\omega^{2}-\omega_{\mathrm{A}}^{2}\right)}\right] \chi=0,
$$

where $\Delta$, defined in Eq. (A1), becomes

$$
\Delta=-\rho^{\prime}\left(r \Omega^{2}-G M_{*} / r^{2}\right)-r \rho \Omega^{2^{\prime}}+r\left(\mu^{2} B_{z}^{2}\right)^{\prime} .
$$

This at once demonstrates the essential difference with the rotating $\theta$ pinch of Sec. IV: for Keplerian rotation, the centrifugal term is exactly cancelled by the gravitational term, so that the first term, representing the Rayleigh-Taylor instability drive, trivially vanishes. Since the last term is negligible $\left(B_{z}^{2} \sim \epsilon^{2} / \beta\right)$, only the shear flow term remains:

$$
\Delta \approx-r \rho \Omega^{2^{\prime}} \equiv-\rho\left(\kappa_{\mathrm{e}}^{2}-4 \Omega^{2}\right),
$$

where $\kappa_{\mathrm{e}}^{2} \equiv\left(1 / r^{3}\right)\left(r^{4} \Omega^{2}\right)^{\prime}$ is the square of the epicyclic frequency. For the equilibrium distributions (41), this yields $\kappa_{\mathrm{e}}=\Omega^{2}$ and $\Delta=3 \rho \Omega^{2}$.

In agreement with the general rationale of the MRIs (to provide a mechanism for turbulent dissipation), the vertical wave number is chosen very large, $k \gg 1$, so that $\omega_{\mathrm{A}} \sim 1$ in the units chosen. This implies that the term $k \mu B_{z}^{2} \sim(1 / k)$ in Eq. (43) may be neglected, with the enormous simplification that $\omega$ only appears squared in the spectral equation, just like in static MHD. The Coriolis shift is then negligible and the eigenfunctions are essentially real, so that the solution path will be the same as what it would be for static equilibria (viz. the real and imaginary axes). With these additional simplifications of Eq. (43), a quadratic form may be constructed,

$$
\int \frac{\rho}{r}\left\{\left(\omega^{2}-\omega_{\mathrm{A}}^{2}\right)\left|\chi^{\prime}\right|^{2}+k^{2}\left[\omega^{2}-\omega_{\mathrm{A}}^{2}-\kappa_{\mathrm{e}}^{2}-\frac{4 \Omega^{2} \omega_{\mathrm{A}}^{2}}{\omega^{2}-\omega_{\mathrm{A}}^{2}}\right]|\chi|^{2}\right\} d r=0,
$$


which immediately yields the well known local instability criterion for MRIs:

$$
\omega_{\mathrm{A}}^{2}\left(\omega_{\mathrm{A}}^{2}+\kappa_{\mathrm{e}}^{2}-4 \Omega^{2}\right)<0, \quad \text { or } \quad 0<k^{2} B_{z}^{2}<\Delta
$$

showing that the magnetic field is necessary for the instability but eventually stabilizes it again. The corresponding expression for the eigenfrequencies of local modes $\chi \sim \exp (\mathrm{i} q r)$,

$$
\omega^{2} \approx \omega_{\mathrm{A}}^{2}+(1 / E)\left[\frac{1}{2} \kappa_{\mathrm{e}}^{2} \pm \frac{1}{2}\left(\kappa_{\mathrm{e}}^{4}+16 E \Omega^{2} \omega_{\mathrm{A}}^{2}\right)^{1 / 2}\right], \quad E \equiv 1+q^{2} / k^{2},
$$

yields estimates of both the growth rate of the most global MRI, for $q^{2} / k^{2} \ll 1$, and the frequencies of the continua, for $q^{2} / k^{2} \gg 1$.

\section{B. Spectral Web results}

Figures 13-15 show the numerically calculated Spectral Web and corresponding eigenfunctions for the MRIs, were none of the above approximations were exploited. In fact, the numerical results exhibit significant differences with respect to compressibility and the Coriolis shift of the eigenvalues. They were obtained by solving the full compressible $(\gamma=5 / 3)$ equations (20) for complex values of $\omega$, substituting the explicit equilibrium profiles (41). The right solutions $\boldsymbol{\xi}^{r}$ were exploited, i.e. the complementary energy $W_{\text {com }}^{\mathrm{r}}$ was evaluated at the inner edge of the disk. The values of $\epsilon, \beta$, and $\mu_{1}$ were chosen according to the ordering (42). However, the radial width $\delta$ was chosen of order unity, rather than much larger than 1 , in order to be able to distinguish the different modes on the solution path. We will see below that extrapolation to larger radial widths, $\delta \gg 1$, can easily be inferred from the results obtained for $\delta \sim 1$.

The Spectral Web shown in Fig. 13 has a very clear structure (note Ref. 32). All modes are located on the solution path (in red). For the unstable modes, that path slightly deviates from the imaginary axis due to the Coriolis shift caused by the non-vanishing 'toroidal' field component $B_{\theta}$, in agreement with earlier MRI calculations. ${ }^{40,41}$ Due to the choice of a moderate value of the radial width $\delta$ of the disk, the conjugate path (in blue) consists of a series of separate 'pancakes', with precisely one genuine (and one false) EV on each pancake, thus determining the sequence number $n(=1, \ldots 29)$ of the unstable modes with growth rates that diminish monotonically as $n$ increases, in agreement with the Complex Oscillation Theorem of Paper 1. This counting is exactly continued by the counting of nodes of the real eigenfunctions of the stable modes $\left(n \geq 30\right.$ and $\left.n^{\prime} \geq 30\right)$ along the real parts of the solution path, where the Real Oscillation Theorem of Ref. 11 dictates monotonicity of the eigenvalue $\omega$ as a function of $n$ outside the genuine and apparent singularity regions. The latter include the collection of Doppler shifts $\omega=\Omega_{0} \equiv m v_{\theta} / r+k v_{z}$ (here degenerate at $\omega=0$ ), at the same frequencies as the HD flow continuum singularities (but no longer representing a continuum in ideal $\mathrm{MHD}^{11}$ ). At $\nu=0$ (but $\sigma=\sigma_{0} \neq 0$ ), the stable modes are split into two sequences, an anti-Sturmian one with modes approaching the backward continua and a Sturmian one approaching the forward continua.

Three representative MRI eigenfunctions are shown in Fig. 14. The first one $(n=1)$, with the maximum growth rate, is clearly localized at the position where the stability criterion (47) is violated most, i.e. at the inner edge of the disk. As $n$ increases, the growth rate $\nu$ decreases and the eigenfunction spreads over the whole disk. For the last unstable mode $(n=29)$, the amplitude of the imaginary part of the eigenfunction has decreased, whereas 
the eigenfunctions of the stable modes $(n \geq 30)$ become real. For the purpose of reference, we report the precise eigenvalues of the lowest $n$ stable modes highlighted in Fig. 13:

$$
\begin{aligned}
& n=30^{\prime}: \quad \sigma=-2.2985 \times 10^{-2}, \quad n=30: \quad \sigma=2.1366 \times 10^{-2} \text {, } \\
& n=31^{\prime}: \quad \sigma=-8.0106 \times 10^{-2}, \quad n=31: \quad \sigma=7.8519 \times 10^{-2} \text {. }
\end{aligned}
$$

The asymmetry of these values with respect to $\sigma=\sigma_{0}$ is due to the Coriolis shift.

For larger values of $n$, the eigenfunctions have an increasing number of oscillations in the region corresponding to the boundaries of the approached continua. This approach is shown for $n=100$ and $n^{\prime}=100$ in the zoomed out Spectral Web of Fig. 15(a), with a plot of the radial dependence of the continua in the upper frame. The eigenfunction for $n=100$ shown in Fig. 15(b) illustrates that, eventually, an infinite number of oscillations occurs at the radial location corresponding to the boundary of the pertinent (forward or backward) slow magneto-sonic continuum, i.e. at the outer edge of the disk. Hence, those boundaries of the continua are cluster points of the discrete spectrum of stable "MRIs" (quotation marks to indicate the contradiction in terminology).

\section{The spectral connection}

It is now evident how the Spectral Web of Figs. 13 and 15 will be affected by choosing $\delta \gg 1$ (i.e. $r_{2} \rightarrow \infty$ ). The continua $\Omega_{\mathrm{A}, \mathrm{S}}^{-}$and $\Omega_{\mathrm{A}, \mathrm{S}}^{-}$will then extend to $\omega=0$, pushing the infinity of cluster modes onto the complex branch of the solution path. The Coriolis shift will vanish at $\omega=0$, which then becomes the branching point of the solution path and a cluster point of infinitely many unstable MRIs. Hence, the solution path in between the most unstable $n=1$ mode and the $n \rightarrow \infty$ modes at the origin will be densely packed (not 'dense' in the mathematical sense though) with discrete modes. However, the fastest growing one will not be affected much since it only depends on the conditions of where it is localized, viz. at the inner edge of the disk. Thus, in peculiar contrast with instabilities of laboratory plasmas (e.g. tokamaks), the lowest $n$ modes with the highest growth rates are here the "local" ones, whereas the higher $n$ modes with much reduced growth rates are the "global" ones spreading over the whole disk.

For finite $\delta$, the division of unstable MRIs and stable modes occurs at the marginal $(\nu=0)$ value of the solution-averaged Doppler-Coriolis shift $\sigma_{0}=\bar{V}\left[\chi\left(\sigma_{0}\right)\right]$, i.e. at the frequency where the solution path intersects the real axis (indicated by the crosses in the side frame of Fig. 13). An explicit expression for $\sigma_{0}$ may be obtained from the general expression (22) by, first, noting that the Doppler part vanishes for the present case (since $m=0$ ), and, next, exploiting the approximations (42) and assuming $\sigma$ to be small to simplify the expressions for the two tangential components of $\boldsymbol{\xi}$ resulting from Eqs. (A6) and (A7):

$$
\mathrm{i} \xi_{\theta} \approx-\frac{2 B_{\theta}}{k r^{2} B_{z}}\left[1+\frac{r^{2} \Lambda}{2 \gamma p}+\frac{r \rho \Omega}{k B_{\theta} B_{z}} \sigma\right] \chi, \quad \mathrm{i} \xi_{z} \approx-(k r)^{-1} \chi^{\prime}
$$

Here, the second term in square brackets involves the deviation $\Lambda$ from Keplerian rotation, which is small but not at all negligible for the present purpose:

$$
\Lambda \equiv \rho\left(\Omega^{2}-G M_{*} / r^{3}\right) \approx-\frac{5}{2 r^{2}} p \sim \epsilon^{2} \quad \Rightarrow \quad \frac{r^{2} \Lambda}{2 \gamma p} \approx-\frac{5}{4 \gamma}
$$


The last term in square brackets corresponds to the term $k \mu B_{z}^{2}$ of Eq. (43) that was also neglected in the analysis of Eqs. (46)-(48). Since this term involves the unknown $\sigma$, the corresponding integral needs to be moved to the LHS of Eq. (22). This yields the following expression for the Coriolis shift:

$$
\sigma_{0} \approx-\frac{4 \mu_{1}}{k}\left(1-\frac{5}{4 \gamma}\right) \int \rho\left(\Omega / r^{2}\right)|\chi|^{2} d r / \int(\rho / r)\left[|\chi|^{2}+\left|\chi^{\prime} / k\right|^{2}+4\left(\Omega^{2} / \omega_{\mathrm{A}}^{2}\right)|\chi|^{2}\right] d r .
$$

This is still an implicit equation for the determination of $\sigma_{0}$ since the integrals involve the solution $\chi\left(\sigma_{0}\right)$ of the spectral equation. However, they may be estimated for the explicit equilibrium (41) by rather straightforward approximations. The corresponding values of $\sigma_{0}$ are in excellent agreement with the results of the numerical code.

The factor $1-5 /(4 \gamma)$, which equals $1 / 4$ for $\gamma=5 / 3$ (but 1 for $\gamma=\infty$ ), produces a large effect of compressibility at marginal stability (as illustrated in the side frame of Fig. 13): $\sigma_{0}=-8.262 \times 10^{-4}$ for the compressible modes, whereas $\sigma_{0}=-3.373 \times 10^{-3}$ for the incompressible ones. A similar large factor of about 4 occurs in the deviation of the solution path from the imaginary axis (illustrated in the main frame of Fig. 13 for the compressible modes). For example, the EVs corresponding to the eigenfunctions of Fig. 14 are given by:

$$
\begin{aligned}
& n=1: \quad \sigma=-2.0312(-8.2883) \times 10^{-3}, \nu=6.2772(6.2077) \times 10^{-1} \\
& n=10: \quad \sigma=-1.2866(-5.2491) \times 10^{-3}, \nu=3.8605(3.8104) \times 10^{-1} \\
& n=29: \quad \sigma=-8.2615(-33.728) \times 10^{-4}, \nu=7.3537(5.9292) \times 10^{-2}
\end{aligned}
$$

where the values in brackets are for incompressibility. The latter assumption gives reasonable estimates for the growth rates, but the real parts of the eigenvalues are completely off.

For finite size disks, the split in unstable and stable modes is of considerable physical significance, since the unstable MRIs are relevant for turbulence studies of the accretion process, whereas the frequencies and eigenfunctions of the stable modes contain important information about the morphology of the disk because of the strong radial dependence of the equilibrium profiles. The latter, stable, part of the spectrum may become relevant in some distant future when the subject of magneto-seismology (correlating spectral studies with observations) of accretion disks ${ }^{8}$ has come of age. As for the present, it appears that we have succeeded to incorporate the MRI into the general MHD spectral theory of equilibria with sizeable background flows by means of the method of the Spectral Web.

\section{SUMMARY}

- The new method of the Spectral Web to calculate the spectrum of waves and instabilities of plasma equilibria with sizeable flows, developed in the preceding Paper $\mathrm{I}^{1}{ }^{1}$ is applied to a collection of classical MHD instabilities operating in cylindrical plasmas with shear flow or rotation. After a review of the basic concepts of the complementary energy giving the solution path and the conjugate path, which together constitute the Spectral Web (Section I), the cylindrical model is presented and the spectral equations are derived (Section II).

- The first example concerns the internal kink instabilities of a cylindrical force-free magnetic field of constant $\alpha$ subjected to a parabolic shear flow profile. The old VoslamberCallebaut $^{14}$ stability diagram and the associated Goedbloed-Hagebeuk ${ }^{15}$ growth rate calculations for static equilibria are replaced by a new intricate stability diagram and associated 
complex growth rates for the stationary model. The power of the Spectral Web method is demonstrated by showing that the two associated paths in the complex $\omega$-plane nearly automatically guide to the new class of Global Alfvén Instabilities (GAIs) of the force-free configuration, that would have been very hard to predict by other methods (Section III).

- The second example concerns the Rayleigh-Taylor instability of a rotating theta-pinch. The old literature ${ }^{25,30}$ is revisited and shown to suffer from inconsistencies that are remedied. The most global $n=1$ instability and a cluster sequence of more local but much more unstable $n=2,3, \ldots \infty$ modes are located on separate solution paths in the HD version of the instability, whereas they merge in the MHD version. The Spectral Web offers visual demonstration of the central position the HD flow continuum and of the MHD Alfvén and slow magneto-sonic continua in the respective spectra by connecting the discrete modes in the complex plane by physically meaningful curves towards the continua (Section IV).

- The third example concerns the magneto-rotational instability (MRI) ${ }^{35,36}$ thought to be operating in accretion disks about black holes ${ }^{37}$. The sequence $n=1,2, \ldots$ of unstable MRIs is located on one continuous solution path, but also on infinitely many separate loops ('pancakes') of the conjugate path with just one MRI on each of them. For narrow accretion disks, those sequences are connected with the slow magneto-sonic continuum, which is far away though from the marginal stability transition. In this case, the Spectral Web method is the first to effectively incorporate the MRIs into the general MHD spectral theory of equilibria with background flows (Section V).

- Together, the three examples provide compelling evidence of the computational power of the Spectral Web Method.

\section{Acknowledgement}

I am indebted to Jeffrey Freidberg and Daniel Barnes for suggesting the investigation of the rotating theta pinch and FRC, and to Rony Keppens for stimulating discussions on the MRIs in accretion disks. 


\section{Appendix A: Coefficients of the spectral differential equations}

It is expedient to renormalize the azimuthal variables with $r$ by redefining $\bar{h}^{2} \equiv r^{2} h^{2}$, $\bar{B}_{\theta} \equiv B_{\theta} / r$ and $\bar{v}_{\theta} \equiv v_{\theta} / r$, and to introduce abbreviations for two equilibrium functions, $\Delta(r)$ and $\Lambda(r)$, and three perturbation functions, $P(r ; \widetilde{\omega}), Q(r ; \widetilde{\omega})$ and $R(r ; \widetilde{\omega})$ :

$$
\begin{aligned}
& \Delta \equiv r\left(\bar{B}_{\theta}^{2}-\rho \bar{v}_{\theta}^{2}\right)^{\prime}+\rho^{\prime} \Phi_{\mathrm{gr}}^{\prime}, \quad \Lambda \equiv \rho\left(\bar{v}_{\theta}^{2}-\Phi_{\mathrm{gr}}^{\prime} / r\right) \\
& P \equiv \bar{B}_{\theta} F+\rho \bar{v}_{\theta} \widetilde{\omega}, \quad Q \equiv \bar{B}_{\theta} F P+\bar{B}_{\theta}^{2} \widetilde{A}, \quad R \equiv \bar{B}_{\theta}^{2}\left[2 m P-\bar{h}^{2}\left(\bar{B}_{\theta}^{2}+\rho \bar{v}_{\theta}^{2}\right)\right] .
\end{aligned}
$$

The coefficients $A, B$ and $C$ of the second order ODE (12) are then defined by

$$
\begin{aligned}
A & \equiv \frac{1}{r}(\widetilde{A}+\Delta) \\
B & \equiv-\frac{4}{r}\left\{\left(\rho \widetilde{\omega}^{2}-k^{2} \gamma p\right) P^{2}-\rho \widetilde{\omega}^{2} R+\left(m \rho \widetilde{\omega}^{2} P-\bar{h}^{2} Q\right) \Lambda+\frac{1}{4} \bar{h}^{2} \widetilde{A} \Lambda^{2}\right\} \\
C & \equiv \frac{2}{r^{2}}\left\{m \widetilde{S} P-r^{2} \rho \widetilde{\omega}^{2}\left(Q-\frac{1}{2} \widetilde{A} \Lambda\right)\right\}
\end{aligned}
$$

whereas the coefficient $E$ of the first order system (20) is defined by

$$
E \equiv-\frac{1}{r^{2}}\left\{\widetilde{A} \widetilde{S}(\widetilde{A}+\Delta)-4 \widetilde{S} P^{2}+4 r^{2}\left(Q-\frac{1}{2} \widetilde{A} \Lambda\right)^{2}\right\}
$$

This corresponds with the coefficients derived by Bondeson et al. ${ }^{7}$ extended with the contributions of the gravitational potential. ${ }^{8}$

The perpendicular and parallel components of $\boldsymbol{\xi}$ follow directly from $\chi \equiv r \xi_{r}$ :

$$
\begin{aligned}
& \eta=\frac{1}{r B D}\left\{G \widetilde{S} \chi^{\prime}-2\left[k \gamma p F P-r\left(\bar{B}_{z} P-G \bar{B}_{\theta}^{2}+\frac{1}{2} G \Lambda\right) \rho \widetilde{\omega}^{2}\right] \chi\right\}, \\
& \zeta=\frac{1}{r B D}\left\{F \gamma p \widetilde{A} \chi^{\prime}+2\left[k \gamma p G P+r\left(\bar{B}_{\theta} P-F \bar{B}_{\theta}^{2}+\frac{1}{2} F \Lambda\right)\left(\rho \widetilde{\omega}^{2}-h^{2} B^{2}\right)\right] \chi\right\},
\end{aligned}
$$

where $G \equiv m B_{z} / r-k B_{\theta}$. The solution-averaged Doppler-Coriolis shift $\bar{V}$ may be obtained by substituting $\mathrm{i} \xi_{\theta} \equiv\left(B_{z} \eta+B_{\theta} \zeta\right) / B$ and $\mathrm{i} \xi_{z} \equiv\left(-B_{\theta} \eta+B_{z} \zeta\right) / B$ into the definition (22).

In the first order formulation (20), for complex frequencies, all expressions split into real and imaginary components:

$$
\left(\begin{array}{c}
\chi_{1}^{\prime} \\
\chi_{2}^{\prime} \\
\Pi_{1}^{\prime} \\
\Pi_{2}^{\prime}
\end{array}\right)+\left(\begin{array}{cccc}
\hat{C}_{1} & -\hat{C}_{2} & \hat{D}_{1} & -\hat{D}_{2} \\
\hat{C}_{2} & \hat{C}_{1} & \hat{D}_{2} & \hat{D}_{1} \\
\hat{E}_{1} & -\hat{E}_{2} & -\hat{C}_{1} & \hat{C}_{2} \\
\hat{E}_{2} & \hat{E}_{1} & -\hat{C}_{2} & -\hat{C}_{1}
\end{array}\right)\left(\begin{array}{l}
\chi_{1} \\
\chi_{2} \\
\Pi_{1} \\
\Pi_{2}
\end{array}\right)=0
$$

where $\hat{C} \equiv C / N, \hat{D} \equiv D / N, \hat{E} \equiv E / N$, with real and imaginary parts $\hat{C}_{1,2}, \hat{D}_{1,2}, \hat{E}_{1,2}$. The explicit expressions for these coefficients are obtained by straightforward expansion of Eqs. (A2)-(A4). Significant simplification occurs in the incompressible limit $(\gamma p \rightarrow \infty)$. 


\section{Appendix B: Boundary condition at the origin}

Numerical implementation of the left BC (13)(a) requires expansion of the coefficients about the singularity $r=0$. This expansion is essentially different for the mode numbers $m \neq 0$ and $m=0$.

(a) For $m \neq 0$, the leading order terms of the contributing coefficients in Eq. (12) read:

$$
N \approx \frac{1}{r} \widetilde{A}_{0} \widetilde{S}_{0}, \quad D \approx-\frac{m^{2}}{r^{2}} \widetilde{S}_{0}, \quad A \approx \frac{1}{r} \widetilde{A}_{0}, \quad C \approx \frac{2 m}{r^{2}}\left(\bar{B}_{\theta} F+\rho \bar{v}_{\theta} \widetilde{\omega}\right)_{0} \widetilde{S}_{0},
$$

so that close to the origin

$$
\chi \approx c r^{|m|}, \quad \Pi \approx \frac{c}{|m|}\left[\widetilde{A}+2 \operatorname{sgn}(m)\left(\bar{B}_{\theta} F+\rho \bar{v}_{\theta} \widetilde{\omega}\right)\right]_{0} r^{|m|},
$$

where the subscript 0 signifies evaluation at $r=0$.

(b) For $m=0$, the leading order terms of the contributing coefficients read:

$$
\begin{aligned}
N & \approx \frac{1}{r} \widetilde{A}_{0} \widetilde{S}_{0}, \quad D \equiv\left(\rho^{2} \widetilde{\omega}^{4}-k^{2} \widetilde{S}\right)_{0}, \quad A \approx \frac{1}{r} \widetilde{A}_{0}, \\
B & \approx-\frac{1}{r}\left[4\left(\rho \widetilde{\omega}^{2}-k^{2} \gamma p\right)\left(\bar{B}_{\theta} F+\rho \bar{v}_{\theta} \widetilde{\omega}\right)^{2}\right]_{0},
\end{aligned}
$$

so that close to the origin

$$
\chi \approx c\left(r^{2}-\frac{1}{8} \alpha r^{4}\right), \quad \Pi \approx-c\left(\frac{2 \widetilde{A} \widetilde{S}}{\rho^{2} \widetilde{\omega}^{4}-k^{2} \widetilde{S}}\right)_{0} .
$$

The contribution with $\alpha$ in $\chi$ is just added to show consistency of the expansion.

For complex frequencies, the above expressions split into real and imaginary parts, again obtained by straightforward expansion.

\section{Appendix C: Singular expansions}

We here restrict the analysis to plane shear flow $\left(v_{\theta}=0\right)$. Indicating the distance to a singular point by $s \equiv x-x_{s}$, where $x=x_{s}$ is the singular point, it is expedient to introduce the local shear Alfvén Mach number, together with its slow critical value:

$$
\left.M \equiv \frac{\Omega_{0}-\Omega_{0}\left(r_{s}\right)}{\omega_{\mathrm{A}}-\omega_{\mathrm{A}}\left(r_{s}\right)} \approx \frac{\Omega_{0}{ }^{\prime}}{\omega_{\mathrm{A}}{ }^{\prime}}\right|_{r_{s}}\left(=-\left.\frac{\sqrt{\rho} v_{z}{ }^{\prime}}{B_{z}\left(\mu^{\prime} / \mu\right)}\right|_{r_{s}}\right),\left.\quad M_{c} \equiv \sqrt{\frac{\gamma p}{\gamma p+B^{2}}}\right|_{r_{s}},
$$

so that $M / M_{c}=\left(\Omega_{0}{ }^{\prime} / \omega_{\mathrm{S}}^{\prime}\right)_{r_{s}}$. (The expression in large brackets is only valid at rational surfaces, $k / m=-\mu$, or $m / n=-q$.) Local trans-Alfvénic transitions are indicated by $M= \pm 1$ and local trans-slow transitions by $M= \pm M_{c}$. We now summarize the results of three relevant expansions of the singular factors of the spectral equation (12). 
(a) Suydam rational surface expansion

At a rational surface (where $\omega_{\mathrm{A}}=\omega_{\mathrm{S}}=0$ ), a confluence of almost all singularities occurs: $\Omega_{\mathrm{A}}^{ \pm}=\Omega_{\mathrm{S}}^{ \pm}=\Omega_{\mathrm{s} 0}^{ \pm}=\Omega_{0}$ at $x=x_{s}$. For stationary equilibria, a local stability criterion is obtained by analyzing the approach to that confluence at $\sigma=\Omega_{0}, \nu=0$, i.e. at the crossing of the solution path with the real axis. The expansions of the singular factors there read: $\widetilde{\omega}^{2}-\omega_{\mathrm{A}}^{2} \approx\left(M^{2}-1\right) \omega_{\mathrm{A}}^{\prime} s^{2}$, and $\widetilde{\omega}^{2}-\omega_{\mathrm{S}}^{2} \approx \widetilde{\omega}^{2}-\omega_{\mathrm{s} 0}^{2} \approx\left(M^{2}-M_{c}^{2}\right) \omega_{\mathrm{A}}{ }^{\prime} s^{2}$. As derived by Hameiri ${ }^{6}$ and Bondeson et al., ${ }^{7}$ the dominant solution $\chi \sim s^{n}$ of Eq. (12) then has a complex exponent $n$, associated with a cluster point of infinitely rapidly oscillating solutions, if the modified Suydam criterion,

$$
S_{\text {mod }} \equiv \frac{1}{1-M^{2}}\left(\frac{p^{\prime}}{r}-\frac{2 B_{\theta}^{2}}{r^{2}} \frac{M_{c}^{2} M^{2}}{M_{c}^{2}-M^{2}}\right)+\frac{1}{8} B_{z}^{2}\left(\frac{\mu^{\prime}}{\mu}\right)^{2}>0 .
$$

is violated. To avoid local instability, one needs to satisfy $(\mathrm{C} 2)$. The derivation clearly breaks down in the neighborhoods of the critical points $M= \pm M_{c}$ and $M= \pm 1$. These points coalesce for incompressible modes $\left(M_{c}^{2}=1\right)$, resulting in a criterion that is not even obviously satisfied in the absence of a pressure gradient (as for the force free field of Sec. III) because of the large negative second term.

(b) Alfvén cluster point expansion

At an extremum of one of the Alfvén continua, where $\Omega_{\mathrm{A}}^{ \pm^{\prime}}\left(x_{s}\right)=0$ so that $M= \pm 1$, the expansion reads: $\sigma-\Omega_{\mathrm{A}}^{ \pm} \approx-\frac{1}{2} \Omega_{\mathrm{A}}^{ \pm \prime} s^{2}$. Again, the exponent $n$ of the dominant solution is complex, and a cluster point of infinitely rapidly oscillating solutions results, if one of the following condition is satisfied:

$$
\begin{aligned}
& \text { either } \quad 0< \pm \frac{1}{4} \omega_{\mathrm{A}} \Omega_{\mathrm{A}}^{ \pm \prime}<Q_{\mathrm{A}}, \quad \text { or } \quad 0> \pm \frac{1}{4} \omega_{\mathrm{A}} \Omega_{\mathrm{A}}^{ \pm \prime}>Q_{\mathrm{A}}, \\
& \text { where } \quad Q_{\mathrm{A}} \equiv \frac{r G^{2}}{B^{2}}\left(\frac{B_{\theta}^{2}}{r^{2}}-\frac{2 B_{\theta} B_{z} F}{r^{2} G}\right)^{\prime}+\frac{4 k^{2} B_{\theta}^{2}}{r^{2}} \frac{1-2 M_{c}^{2}}{1-M_{c}^{2}} .
\end{aligned}
$$

These solutions correspond to a sequence of stable Global Alfvén Eigenmodes (GAEs), if the cluster point is not overlapped by the rest of the continua.

(c) Slow cluster point expansion

At an extremum of one of the slow continua, where $\Omega_{\mathrm{S}}^{ \pm \prime}\left(x_{s}\right)=0$ so that $M= \pm M_{c}$, the similar expansion gives rise to the conditions for a slow cluster point:

$$
\begin{aligned}
& \text { either } \quad 0< \pm \frac{1}{4} \rho \omega_{\mathrm{S}} \Omega_{\mathrm{S}}^{ \pm \prime \prime}<Q_{\mathrm{S}}, \quad \text { or } \quad 0> \pm \frac{1}{4} \rho \omega_{\mathrm{S}} \Omega_{\mathrm{S}}^{ \pm \prime}>Q_{\mathrm{S}} \\
& \text { where } \quad Q_{\mathrm{S}} \equiv-\frac{M_{c}^{4}}{B^{2}}\left[F^{4}\left(1-M_{c}^{2}\right)+r F^{2}\left(\frac{B_{\theta}^{2}}{r^{2}}\right)^{\prime}-\frac{4 k^{2} B_{\theta}^{2} B^{2}}{r^{2}\left(1-M_{c}^{2}\right)}\right] .
\end{aligned}
$$

A sequence of stable Global Slow Eigenmodes results if the continua do not overlap.

The GAEs, and their prospect of low frequency plasma heating, were described by Appert et $a .^{20}$ for static equilibria. The Alfvén and slow cluster conditions were presented in the form (C3) and (C4) for the static case (where $\pm \Omega_{\mathrm{A}, \mathrm{S}}^{ \pm}{ }^{\prime \prime} \rightarrow \omega_{\mathrm{A}, \mathrm{S}}{ }^{\prime \prime}$ ) by Goedbloed. ${ }^{21}$ The revised expansion needed for incompressible modes $\left(M_{c}^{2}=1\right)$ is described in Sec. IIIC. 
1 J. P. Goedbloed, Phys. Plasmas, to appear.

2 W. A. Newcomb, Ann. Phys. (New York) 10 (1960), 232.

3 E. Frieman and M. Rotenberg, Rev. Modern Physics 32, 898 (1960).

4 I. B. Bernstein, E. A. Frieman, M. D. Kruskal and R. M. Kulsrud, Proc. Roy. Soc. (London) A244, 17 (1958).

5 J. P. Goedbloed, Phys. Plasmas 16, 122110 (2009); Phys. Plasmas 16, 122111 (2009).

6 E. Hameiri, J. Math. Phys. 22, 2080 (1981).

7 A. Bondeson, R. Iacono and A. H. Bhattacharjee, Phys. Fluids 30, 2167 (1987).

8 R. Keppens, F. Casse and J. P. Goedbloed, Astrophys. J. 569, L121(2002).

9 J. P. Goedbloed, R. Keppens and S. Poedts, Advanced Magnetohydrodynamics (Cambridge University Press, Cambridge, 2010), p. 95.

10 J. P. Goedbloed and P. H. Sakanaka, Phys. Fluids 17, 908 (1974).

11 J. P. Goedbloed, A. J. C. Beliën, B. van der Holst and R. Keppens, Phys. Plasmas 11, 4332 (2004).

12 M. van Noort, A. Lagg, S. K. Tiwari and S. K. Solanki, Astron. \& Astrophys. 557, A24 (2013).

13 L. Woltjer, Astrophys. J. 128, 384 (1958).

14 D. Voslamber and D. K. Callebaut, Phys. Rev. 128, 2016 (1962).

15 J. P. Goedbloed and H. J. L. Hagebeuk, Phys. Fluids 15, 1090 (1972).

16 J. B. Taylor, Rev. Mod. Phys. 58, 741 (1986).

17 R. Ortolani and D. Schnack, Magnetohydrodynamics of Plasma Relaxation (World Scientific, Singapore, 1993).

18 P. M. Bellan, Spheromaks (Imperial College Press, London, 2010).

19 T. Wiegelmann and T. Sakurai, Living Rev. Solar Phys. 9, 5 (2012).

20 K. Appert, R. Gruber, F. Troyon and H. J. L. Vaclavik, Plasma Physics 24, 1147 (1982).

21 J. P. Goedbloed, Physica 12D, 107 (1984).

22 C. M. Bender and S. A. Orszag, Advanced Mathematical Methods for Scientists and Engineers (McGraw-Hill, New York, 1978), p. 63.

23 B. van der Holst, A. J. C. Beliën and J. P. Goedbloed, Phys. Rev. Lett. 84, 2865 (2000); Phys. Plasmas 7, 4208 (2000).

24 W. W. Heidbrink, Phys. Plasmas 15, 055501 (2008).

25 J. P. Freidberg and J. A. Wesson, Phys. Fluids 13, 1117 (1970).

26 J. P. Freidberg and L. H. Pearlstein, Phys. Fluids 21, 1207 (1978).

27 D. C. Barnes, Phys. Plasmas 8, 4856 (2001).

28 L. C. Steinhauer, Phys. Plasmas 18, 070501 (2011).

29 M. N. Rosenbluth, N. A. Krall and N. Rostoker, Nucl. Fusion Suppl. I, 143 (1962).

30 J. B. Taylor, Plasma Physics (J. Nucl. Energy Part C) 4, 401 (1962).

31 E. Hameiri, Phys. Fluids 22, 89 (1979).

32 The Figures 9 and 13 were used before, in the different context of illustrating the difference between laboratory and astrophysical plasmas with respect to MHD stability, in:

J. P. Goedbloed, Plasma Physics and Controlled Fusion 60, 014001 (2017).

33 K. M. Case, Phys. Fluids 3, 143 (1960).

34 G. O. Spies, Phys. Fluids 21, 580 (1978).

35 E. P. Velikhov, Soviet Phys.--JETP Lett. 36 (1959) 995. 
36 S. Chandrasekhar, Proc. Nat. Acad. Sci. USA 46 (1960), 253.

37 S. A. Balbus and J. F. Hawley, Astron. J. 376(1991) 214.

38 H. C. Spruit, T. Matsuda, M. Inoue and K. Sawada, Monthly Not. Roy. Astron. Soc. 229 (1987) 517.

39 N. I. Shakura and R. A. Sunyaev, Astron. Astrophys. 24 (1973) 337.

40 B. Dubrulle and E. Knobloch, Astron. Astrophys. 274, 667 (1993).

41 J. W. S. Blokland, E. van der Swaluw, R. Keppens and J. P. Goedbloed, Astron. Astrophys. 444, 337 (2005). 


\section{FIGURE CAPTIONS}

Figure 1. Stability diagram for the $m=1$ internal kink modes of a static equilibrium with a force-free magnetic field of constant $\alpha$. The instability regions of the resistive tearing modes are bound by the dashed curves and shaded black, the instability regions of the ideal MHD modes are bound by the drawn black curves and the thin horizontal red lines and shaded red.

Figure 2. Marginal modes (left) and corresponding real eigenfunctions (right) of the internal kink instabilities of a static equilibrium with a force-free magnetic field: (a) $\alpha a=$ $5.0, \quad k / \alpha=0.16: \nu=4.3171 \times 10^{-2}($ case A); (b) $\alpha a=8.0, k / \alpha=0.05: \nu=1.7766 \times$ $10^{-2}$ (case C, $n=1$ mode); (c) $\alpha a=8.0, \quad k / \alpha=0.05: \nu=1.9294 \times 10^{-3}$ (case $\mathrm{C}, n=2$ mode). The red dashed lines indicate the locations of the rational surfaces.

Figure 3. Spectral Web for the $n=1$ internal kink mode of a force-free magnetic field with shear flow, $v_{z}=1-x^{2}, \alpha a=5.0, k / \alpha=0.16, x_{\text {mix }}=0.3$ (case A). The upper part of the diagram shows the radial profiles of the continua $\Omega_{\mathrm{A}}^{+}$and $\Omega_{\mathrm{A}}^{-}$(in red) and the Doppler frequency $\Omega_{0}$ (dashed).

Figure 4. (a) Complex eigenfunctions of the $n=1$ internal kink instability of a force-free magnetic field with shear flow, $v_{z}=1-x^{2}, \alpha a=5.0$ corresponding to Fig. 3. (a) $k / \alpha=0.16$ : $\sigma=0.47831, \nu=8.7428 \times 10^{-2}$ (case A); (b) $k / \alpha=0.4: \sigma=1.2675, \nu=1.0964 \times 10^{-3}$ (case B). The position of the rational surface is indicated by the red dashed vertical line.

Figure 5. Spectral Web for the $n=1$ and $n=2$ internal kink modes and the first Global Alfvén Instability of a force-free magnetic field with shear flow, $v_{z}=0.9\left(1-x^{2}\right), \alpha a=8.0$, $k / \alpha=0.16, x_{\text {mix }}=0.3$ (case D). The inset shows the logarithmic Spectral Web for some of the higher Global Alfvén Instabilities. The upper part of the diagram shows the radial profiles of the continua $\Omega_{\mathrm{A}}^{+}$and $\Omega_{\mathrm{A}}^{-}$(in red) and the Doppler frequency $\Omega_{0}$ (dashed).

Figure 6. Complex eigenfunctions of the $n=1$ and $n=2$ internal kink instabilities of a force-free magnetic field with shear flow corresponding to Fig. 5, $v_{z}=0.9\left(1-x^{2}\right), \alpha a=8.0$. $k / \alpha=0.16$ (case D). (a) $n=1$ (point $\mathrm{I}_{1}$ in Fig. 5): $\sigma=0.95712, \nu=7.9930 \times 10^{-2}$; (b) $n=2$ (point $\mathrm{I}_{2}$ in Fig. 5 ): $\sigma=0.58027, \nu=9.1377 \times 10^{-2}$. The red dashed lines indicate the rational surfaces.

Figure 7. Stable and corresponding unstable Global Alfvén Eigenmodes of a force-free magnetic field with shear flow corresponding to Fig. $5, v_{z}=0.9\left(1-x^{2}\right), \alpha a=8.0, k / \alpha=0.16$ (case D). (a) First stable Global Alfvén Eigenmode on the interval $(0.8,1.0): \sigma=0.65749$; (b) Third stable Global Alfvén Eigenmode on the interval $(0.8,1.0): \sigma=0.65722$; (c) First Global Alfvén Instability on the complete interval (point $\mathrm{G}_{1}$ in Fig. 5): $\sigma=0.66428, \nu=$ $4.6809 \times 10^{-3}$; (d) Third Global Alfvén Instability on the complete interval (point $\mathrm{G}_{3}$ in Fig. 5): $\sigma=0.65725, \nu=2.7796 \times 10^{-6}$.

Figure 8. Equilibrium density (black) and magnetic field (red) of a rotating theta pinch for $\beta=0.6944$ and $\Omega=1.0542$ (corresponding to $x_{0}=0, \alpha=2, \delta=0.1667$ ). The local HD stability criterion $C_{\text {stab }}$ is indicated in green for compressible (drawn) and incompressible (dashed) plasmas.

Figure 9. Spectral Web consisting of solution path (red) and conjugate path (blue), with some eigenvalues highlighted by black dots, for the $m=1, k=0$ (HD) modes of the rotating theta pinch equilibrium of Fig. 8. The side frame shows the cluster sequence toward the HD flow continuum (red dot in the main frame), with a zoom of the $n=53, \ldots 56$ eigenvalues in the bottom frame. 
Figure 10. The first two (global) eigenfunctions, and a high $n$ (local) one, for the rotating theta pinch equilibrium of Fig. 8: (a) $n=1$ ( $\sigma=0.06547, \nu=0.25248)$, (b) $n=2$ $(\sigma=0.82729, \nu=0.41698),(\mathrm{c}) n=54(\sigma=1.0538, \nu=0.01939)$.

Figure 11. Spectral Web consisting of solution path (red) and conjugate path (blue), with some eigenvalues highlighted by black dots, for the $m=1, k=0.1$ (MHD) modes of the rotating theta pinch equilibrium of Fig. 1. The range of the continua is indicated by grey strips above (Alfvén) and below (slow) the horizontal axis, their spatial dependence is shown in the top frame. The side frame shows a zoom of the unstable $n=5, \ldots 13$ eigenvalues and the stable $n \geq 14$ eigenvalues (only the $n=14$ ones are highlighted).

Figure 12. Growth rate $\nu \equiv \operatorname{Im}(\omega)$ (in red) and Coriolis shift $\widetilde{\sigma} \equiv \operatorname{Re}(\omega)-m \Omega$ (in black) as a function of $k$ for the compressible (drawn) and incompressible (dashed) $m=1, n=1$ modes of the rotating theta pinch equilibrium of Fig. 8. The blue and green curves refer to $\nu$ and $\widetilde{\sigma}$ for the $n=2$ modes. The vertical red and blue dashes indicate the marginal stability transitions.

Figure 13. Spectral Web of MRIs for an accretion disk equilibrium with $\epsilon=0.1, \beta=100$, $\mu_{1}=1, \delta=2$, and mode numbers $m=0, k=70$. The eigenvalues of the $n=1-29$ unstable modes and of the $n=30,31$ and $n=30^{\prime}, 31^{\prime}$ stable modes are highlighted by black dots. The side frame shows a zoom of the Spectral Web close to the origin with the marginal Coriolis shift $\sigma_{0}$ indicated by the cross (the dashed curves refer to incompressible modes).

Figure 14. Eigenfunctions of MRIs corresponding to some of the eigenvalues of the Spectral Web shown in Fig. 13: (a) $n=1$, (b) $n=10$, (c) $n=29$.

Figure 15. (a) Zoomed out lower part of the Spectral Web of Fig. 13, highlighting the continua and the stable $n=100^{\prime}(\sigma=-0.43682)$ and $n=100(\sigma=0.43632)$ modes. The range of the continua is indicated by grey strips above (Alfvén) and below (slow) the horizontal axis, their spatial dependence is shown in the top frame. (b) Eigenfunction of the $n=100$ mode. 


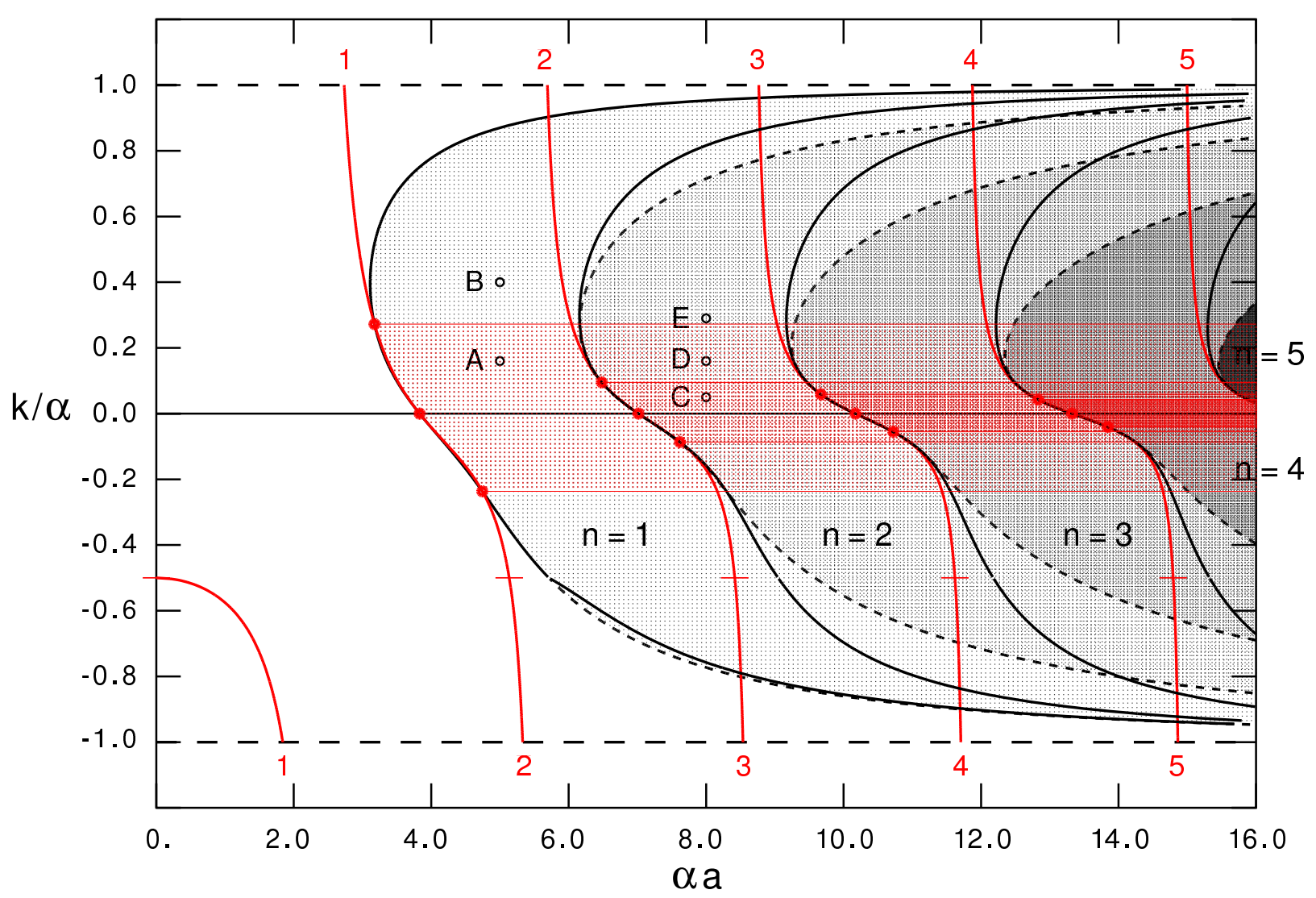

FIG. 1: Stability diagram for the $m=1$ internal kink modes of a static equilibrium with a force-free magnetic field of constant $\alpha$. The instability regions of the resistive tearing modes are bound by the dashed curves and shaded black, the instability regions of the ideal MHD modes are bound by the drawn black curves and the thin horizontal red lines and shaded red. 

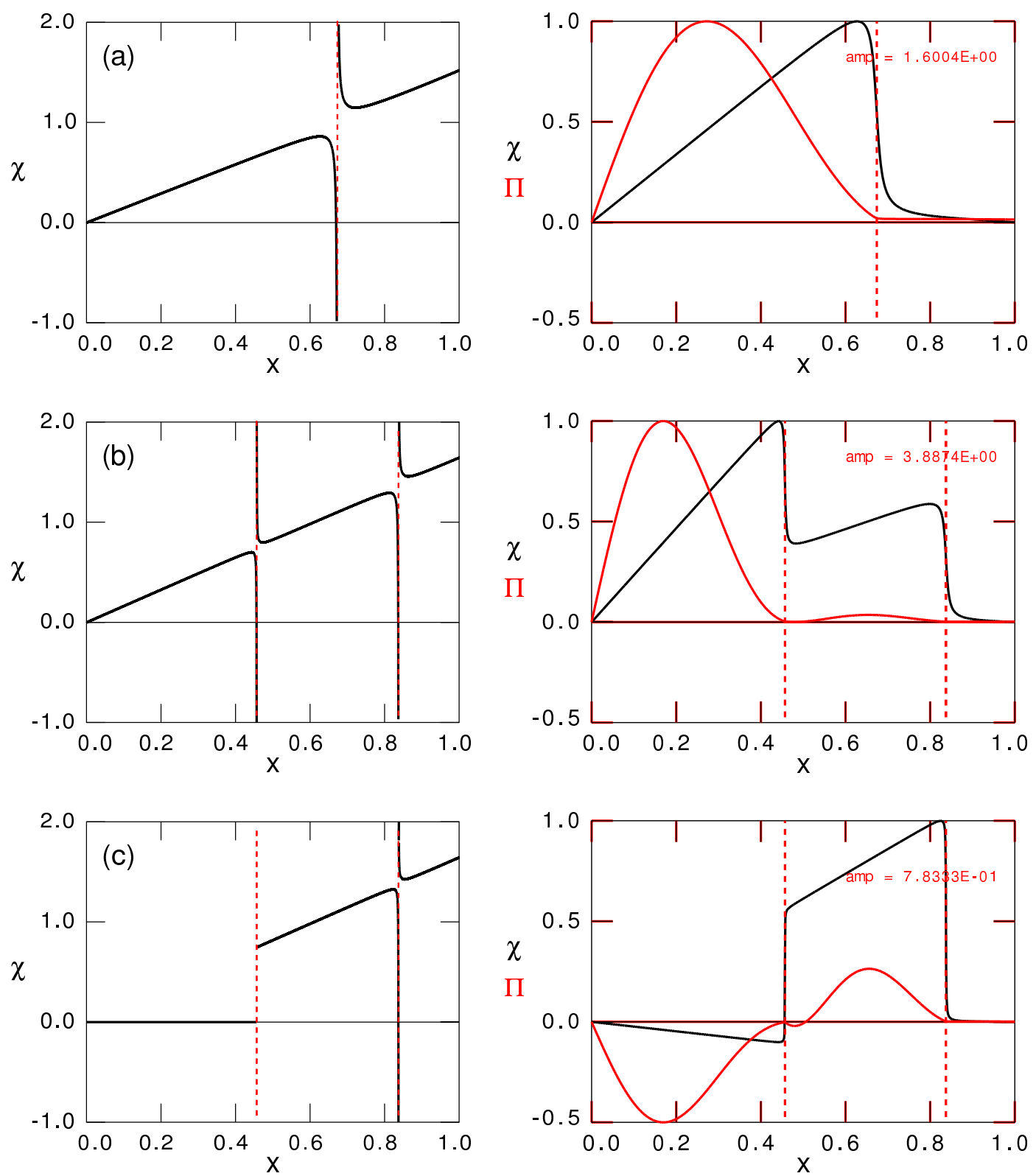

FIG. 2: Marginal modes (left) and corresponding real eigenfunctions (right) of the internal kink instabilities of a static equilibrium with a force-free magnetic field: (a) $\alpha a=5.0, k / \alpha=0.16$ : $\nu=4.3171 \times 10^{-2}$ (case A); (b) $\alpha a=8.0, k / \alpha=0.05: \nu=1.7766 \times 10^{-2}$ (case C, $n=1$ mode); (c) $\alpha a=8.0, \quad k / \alpha=0.05: \nu=1.9294 \times 10^{-3}$ (case $\mathrm{C}, n=2$ mode). The red dashed lines indicate the locations of the rational surfaces. 

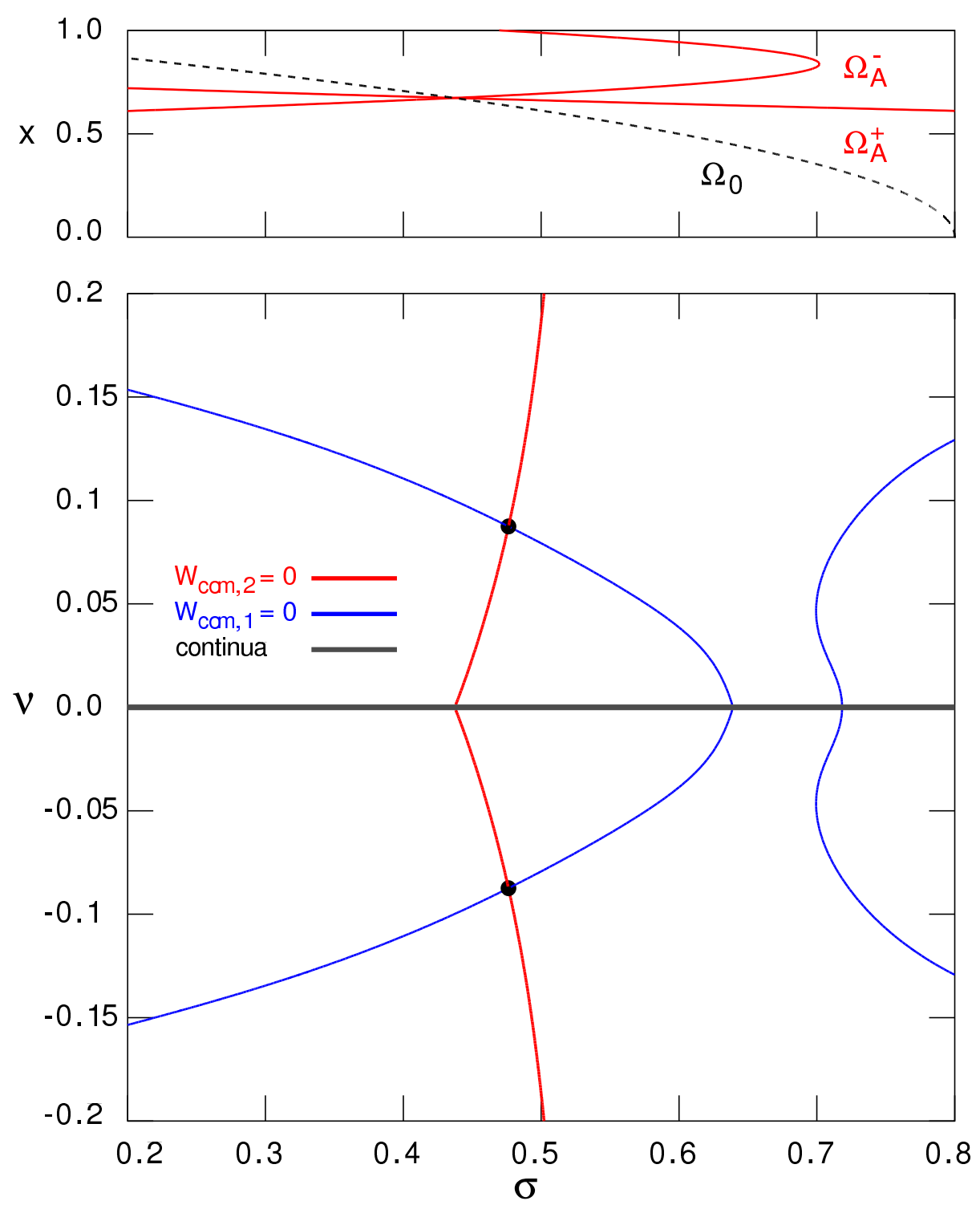

FIG. 3: Spectral Web for the $n=1$ internal kink mode of a force-free magnetic field with shear flow, $v_{z}=1-x^{2}, \alpha a=5.0, k / \alpha=0.16, x_{\text {mix }}=0.3$ (case A). The upper part of the diagram shows the radial profiles of the continua $\Omega_{\mathrm{A}}^{+}$and $\Omega_{\mathrm{A}}^{-}$(in red) and the Doppler frequency $\Omega_{0}$ (dashed). 

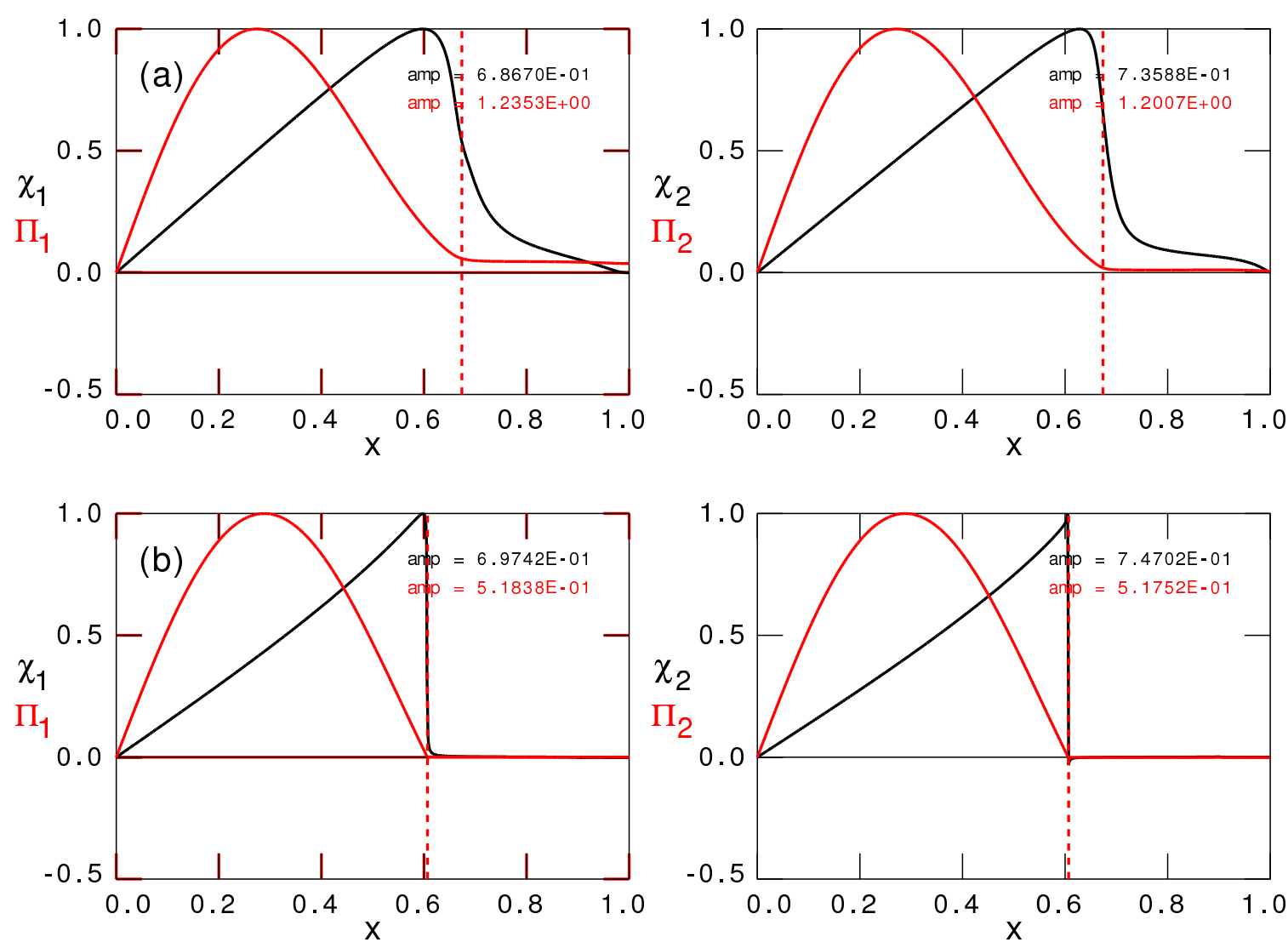

FIG. 4: (a) Complex eigenfunctions of the $n=1$ internal kink instability of a force-free magnetic field with shear flow, $v_{z}=1-x^{2}, \alpha a=5.0$ corresponding to Fig. 3. (a) $k / \alpha=0.16: \sigma=0.47831$, $\nu=8.7428 \times 10^{-2}$ (case A); (b) $k / \alpha=0.4: \sigma=1.2675, \nu=1.0964 \times 10^{-3}$ (case B). The position of the rational surface is indicated by the red dashed vertical line. 

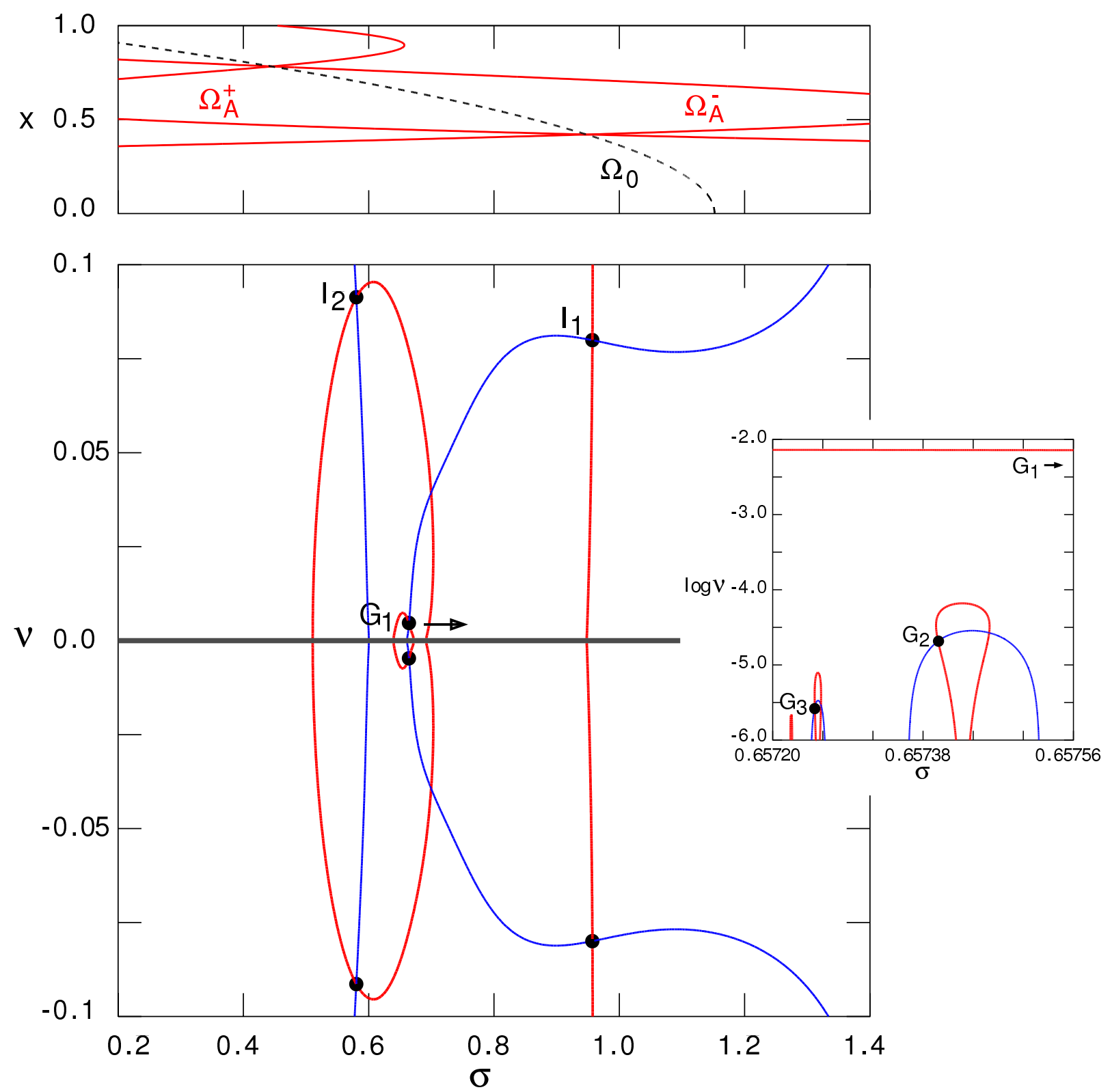

FIG. 5: Spectral Web for the $n=1$ and $n=2$ internal kink modes and the first Global Alfvén Instability of a force-free magnetic field with shear flow, $v_{z}=0.9\left(1-x^{2}\right), \alpha a=8.0, k / \alpha=0.16$, $x_{\text {mix }}=0.3$ (case D). The inset shows the logarithmic Spectral Web for some of the higher Global Alfvén Instabilities. The upper part of the diagram shows the radial profiles of the continua $\Omega_{\mathrm{A}}^{+}$ and $\Omega_{\mathrm{A}}^{-}$(in red) and the Doppler frequency $\Omega_{0}$ (dashed). 

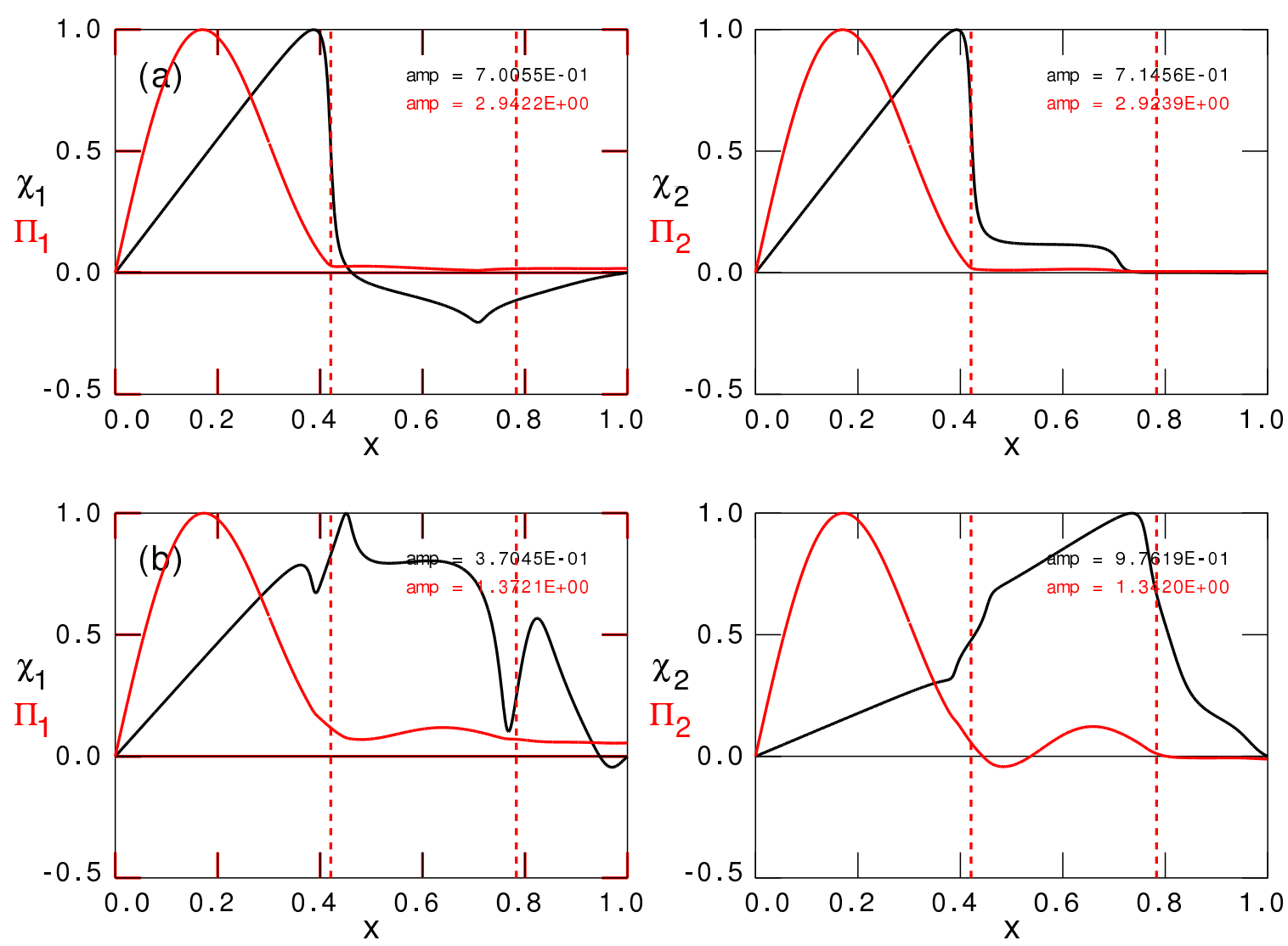

FIG. 6: Complex eigenfunctions of the $n=1$ and $n=2$ internal kink instabilities of a force-free magnetic field with shear flow corresponding to Fig. $5, v_{z}=0.9\left(1-x^{2}\right), \alpha a=8.0 . k / \alpha=0.16$ (case D). (a) $n=1$ (point $\mathrm{I}_{1}$ in Fig. 5): $\sigma=0.95712, \nu=7.9930 \times 10^{-2}$; (b) $n=2$ (point $\mathrm{I}_{2}$ in Fig. 5): $\sigma=0.58027, \nu=9.1377 \times 10^{-2}$. The red dashed lines indicate the rational surfaces. 

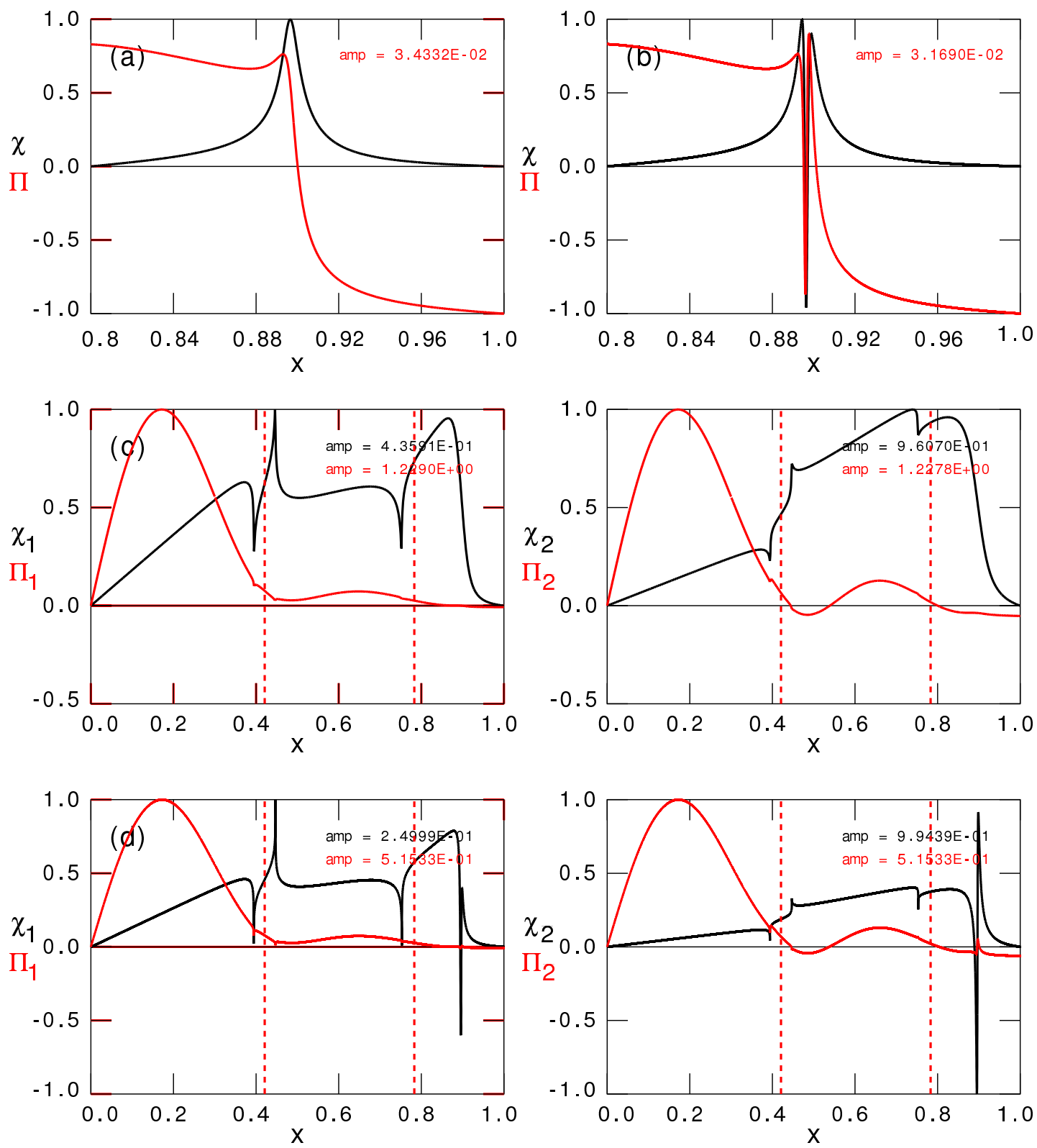

FIG. 7: Stable and corresponding unstable Global Alfvén Eigenmodes of a force-free magnetic field with shear flow corresponding to Fig. $5, v_{z}=0.9\left(1-x^{2}\right), \alpha a=8.0, k / \alpha=0.16$ (case D). (a) First stable Global Alfvén Eigenmode on the interval $(0.8,1.0): \sigma=0.65749$; (b) Third stable Global Alfvén Eigenmode on the interval $(0.8,1.0): \sigma=0.65722$; (c) First Global Alfvén Instability on the complete interval (point $\mathrm{G}_{1}$ in Fig. 5): $\sigma=0.66428, \nu=4.6809 \times 10^{-3}$; (d) Third Global Alfvén Instability on the complete interval (point $\mathrm{G}_{3}$ in Fig. 5 ): $\sigma=0.65725, \nu=2.7796 \times 10^{-6}$. 


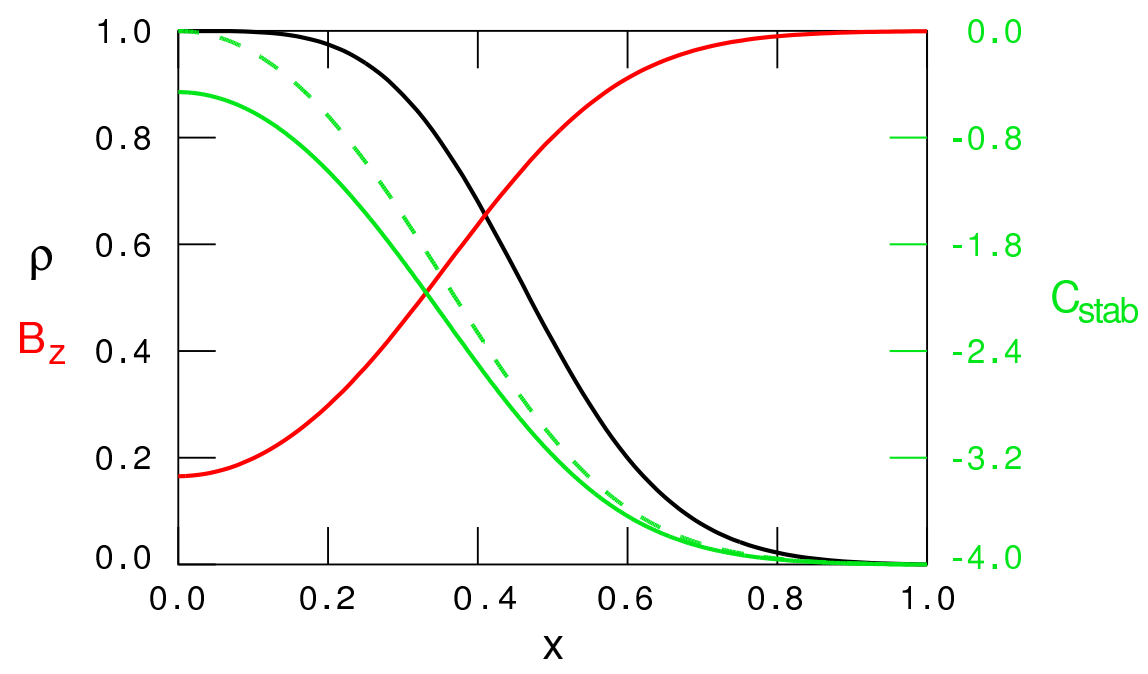

FIG. 8: Equilibrium density (black) and magnetic field (red) of a rotating theta pinch for $\beta=0.6944$ and $\Omega=1.0542$ (corresponding to $x_{0}=0, \alpha=2, \delta=0.1667$ ). The local HD stability criterion $C_{\text {stab }}$ is indicated in green for compressible (drawn) and incompressible (dashed) plasmas.
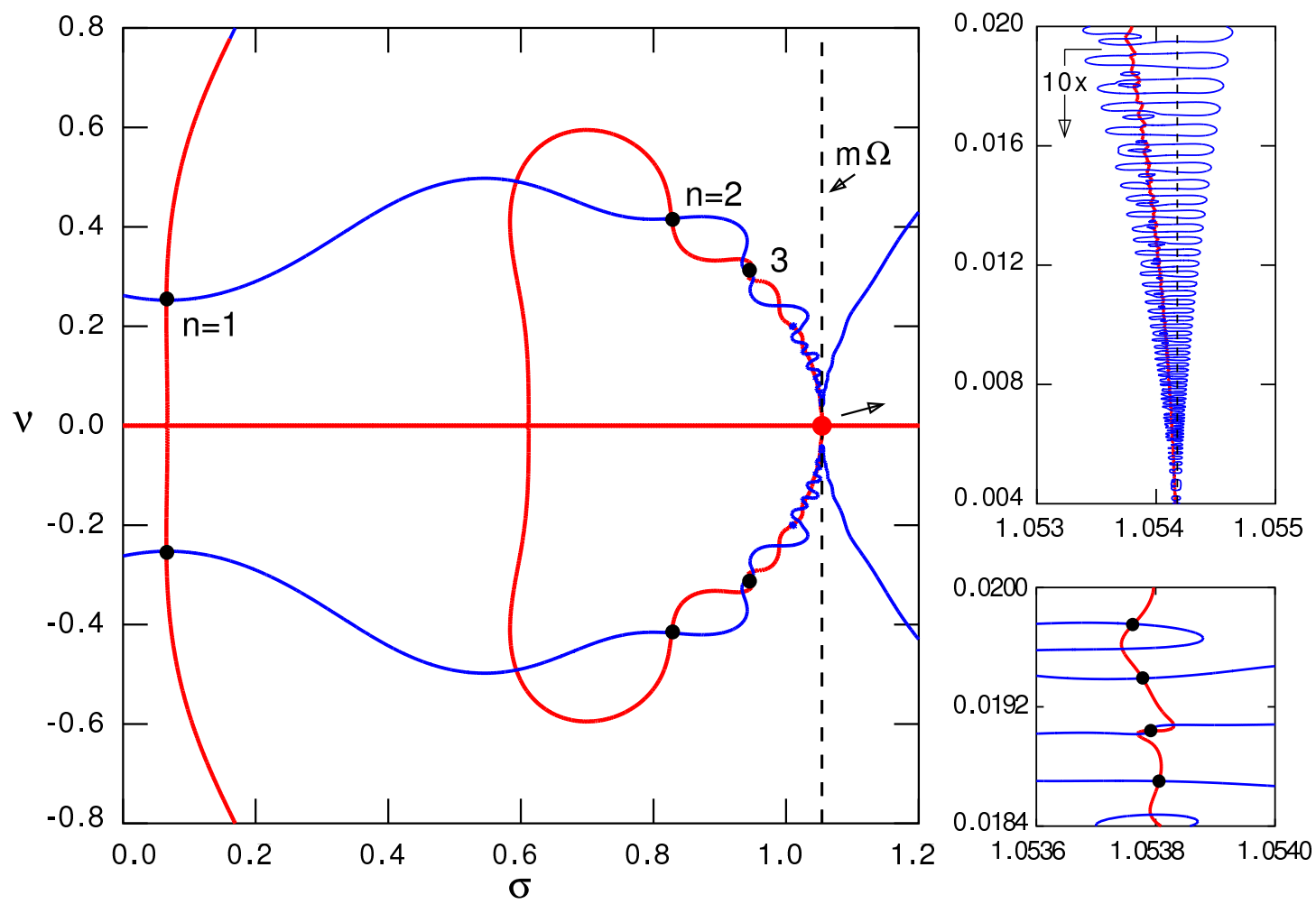

FIG. 9: Spectral Web consisting of solution path (red) and conjugate path (blue), with some eigenvalues highlighted by black dots, for the $m=1, k=0$ (HD) modes of the rotating theta pinch equilibrium of Fig. 8. The side frame shows the cluster sequence toward the HD flow continuum (red dot in the main frame), with a zoom of the $n=53, \ldots 56$ eigenvalues in the bottom frame. 

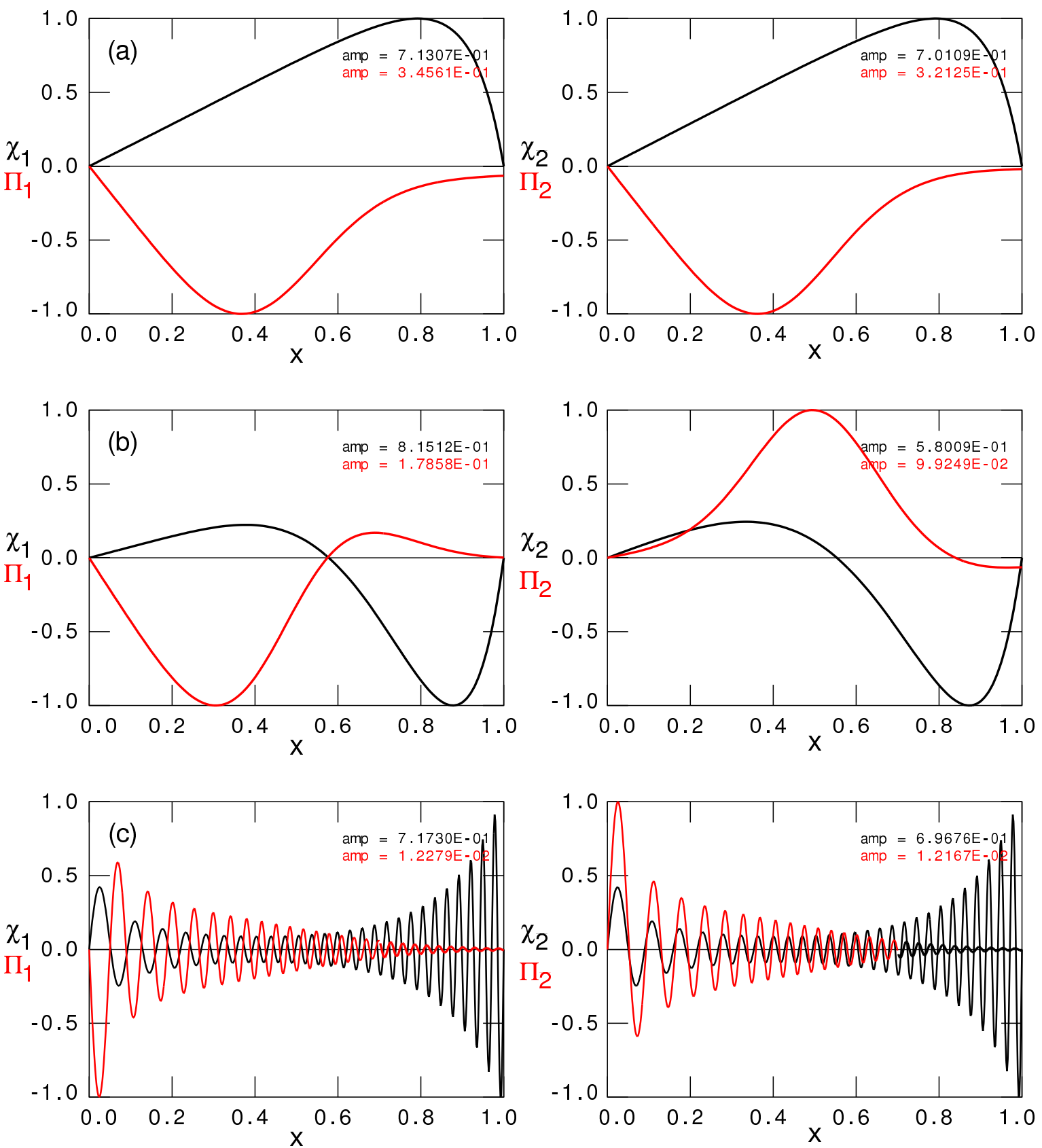

FIG. 10: The first two (global) eigenfunctions, and a high $n$ (local) one, for the rotating theta pinch equilibrium of Fig. 8: (a) $n=1(\sigma=0.06547, \nu=0.25248)$, (b) $n=2(\sigma=0.82729, \nu=0.41698)$, (c) $n=54(\sigma=1.0538, \nu=0.01939)$. 

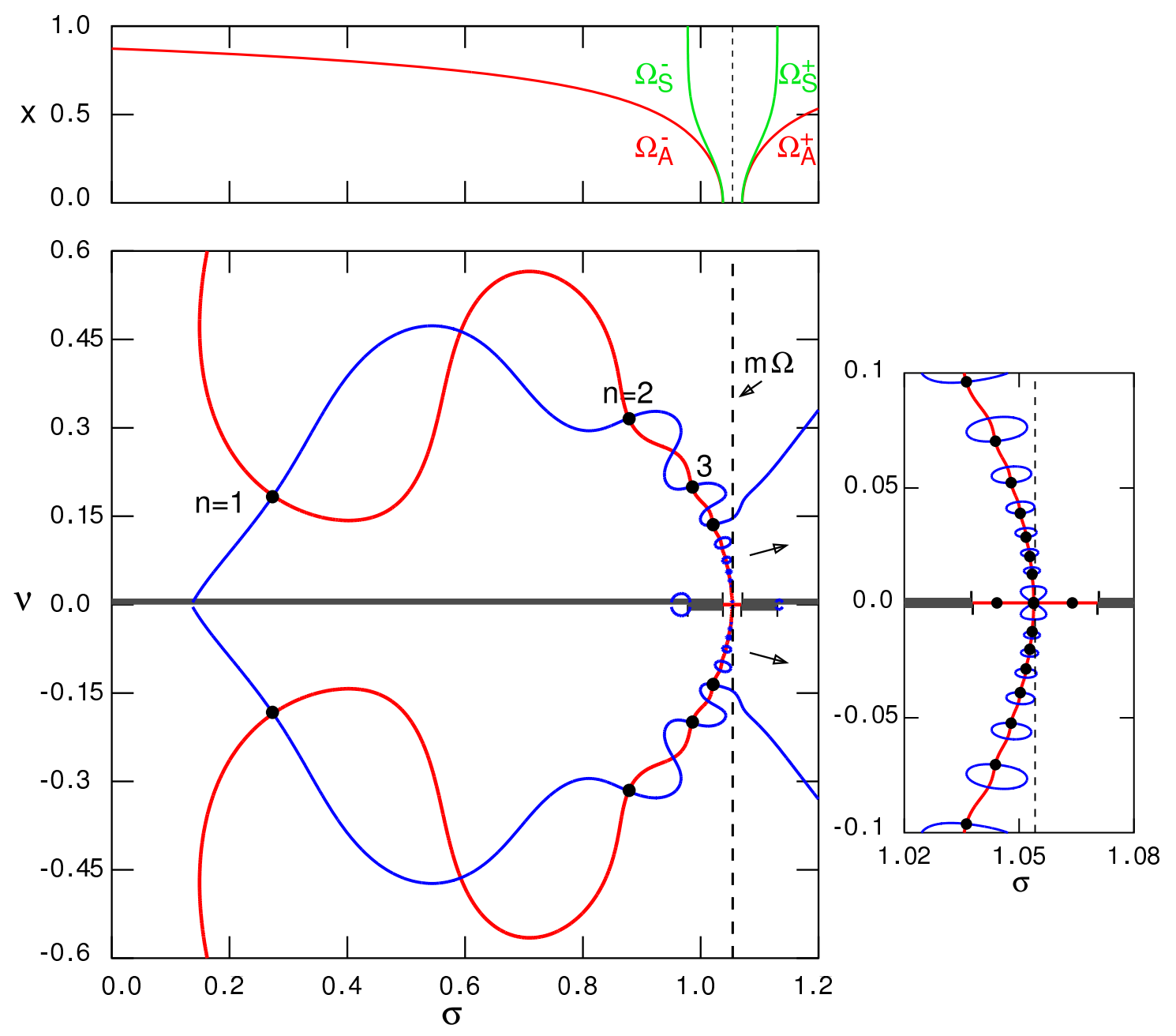

FIG. 11: Spectral Web consisting of solution path (red) and conjugate path (blue), with some eigenvalues highlighted by black dots, for the $m=1, k=0.1$ (MHD) modes of the rotating theta pinch equilibrium of Fig. 8. The range of the continua is indicated by grey strips above (Alfvén) and below (slow) the horizontal axis, their spatial dependence is shown in the top frame. The side frame shows a zoom of the unstable $n=5, \ldots 13$ eigenvalues and the stable $n \geq 14$ eigenvalues (only the $n=14$ ones are highlighted). 


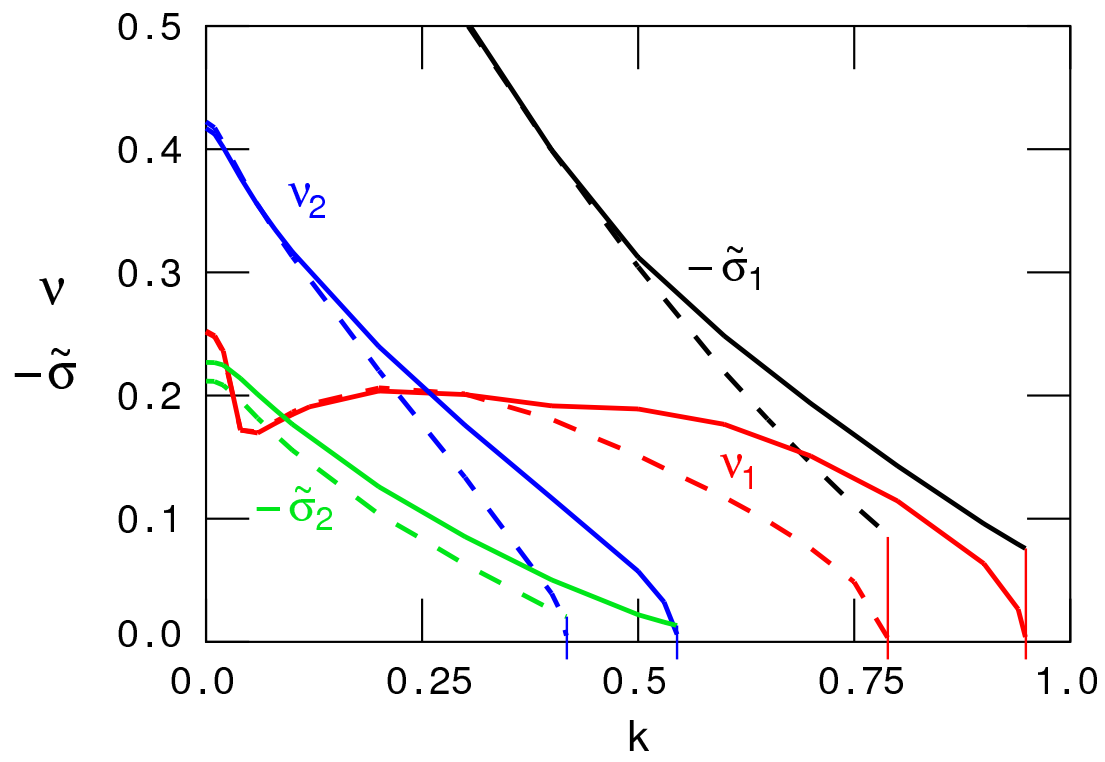

FIG. 12: Growth rate $\nu \equiv \operatorname{Im}(\omega)$ (in red) and Coriolis shift $\widetilde{\sigma} \equiv \operatorname{Re}(\omega)-m \Omega$ (in black) as a function of $k$ for the compressible (drawn) and incompressible (dashed) $m=1, n=1$ modes of the rotating theta pinch equilibrium of Fig. 8. The blue and green curves refer to $\nu$ and $\widetilde{\sigma}$ for the $n=2$ modes. The vertical red and blue dashes indicate the marginal stability transitions. 

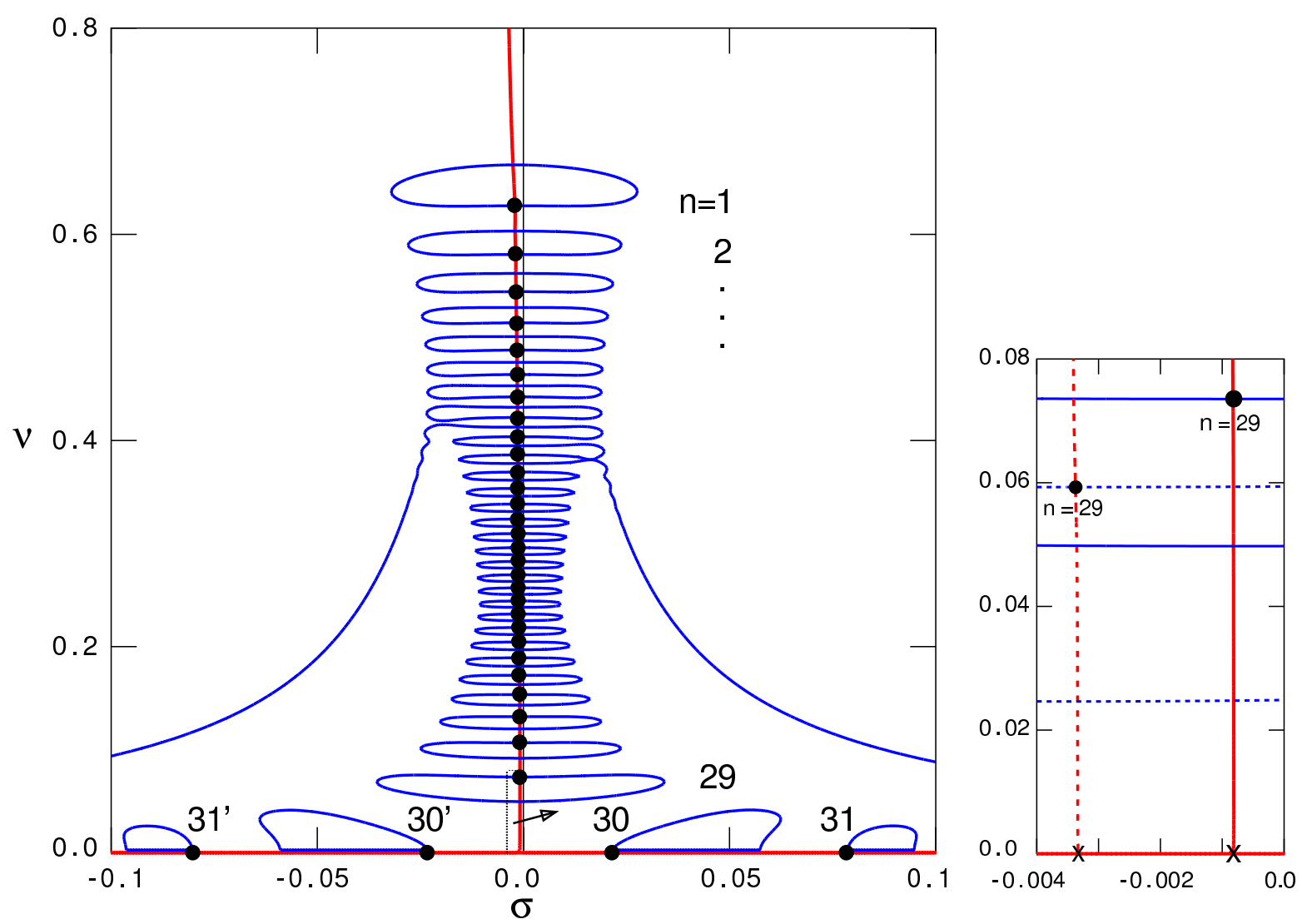

FIG. 13: Spectral Web of MRIs for an accretion disk equilibrium with $\epsilon=0.1, \beta=100, \mu_{1}=1$, $\delta=2$, and mode numbers $m=0, k=70$. The eigenvalues of the $n=1-29$ unstable modes and of the $n=30,31$ and $n=30^{\prime}, 31^{\prime}$ stable modes are highlighted by black dots. The side frame shows a zoom of the Spectral Web close to the origin with the marginal Coriolis shift $\sigma_{0}$ indicated by the cross (the dashed curves refer to incompressible modes). 

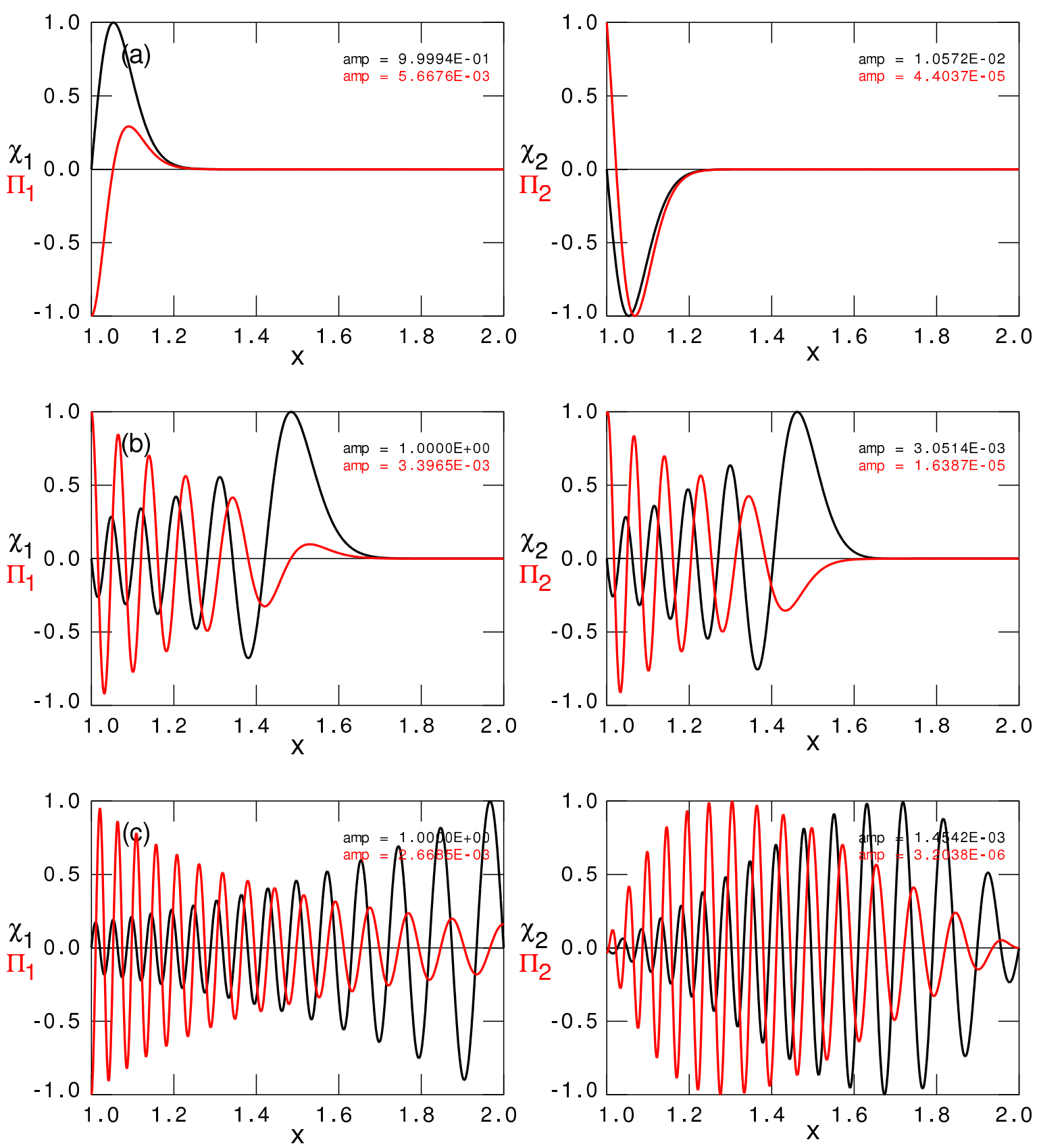

FIG. 14: Eigenfunctions of MRIs corresponding to some of the eigenvalues of the Spectral Web shown in Fig. 13: (a) $n=1$, (b) $n=10$, (c) $n=29$. 

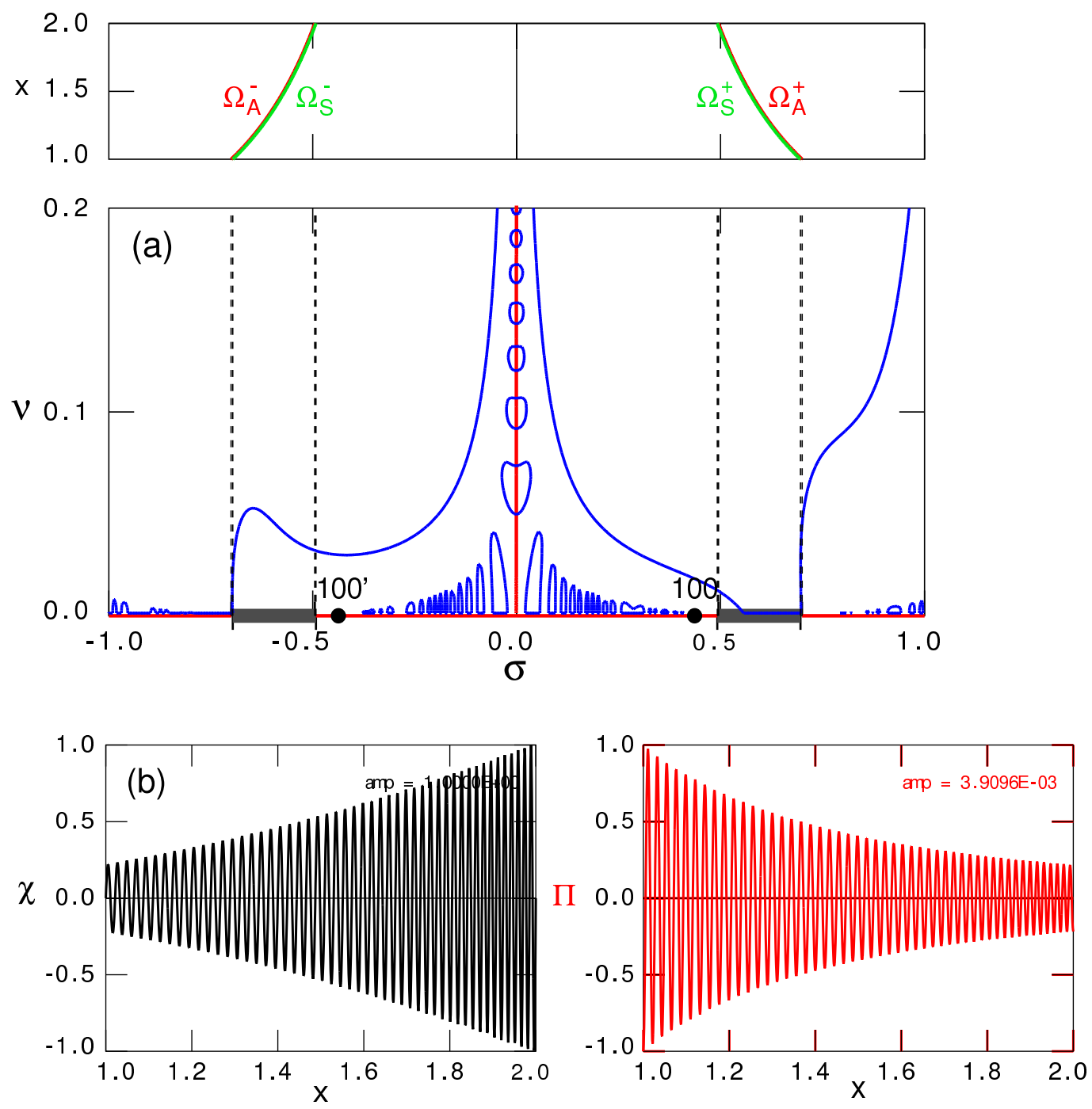

FIG. 15: (a) Zoomed out lower part of the Spectral Web of Fig. 13, highlighting the continua and the stable $n=100^{\prime}(\sigma=-0.43682)$ and $n=100(\sigma=0.43632)$ modes. The range of the continua is indicated by grey strips above (Alfvén) and below (slow) the horizontal axis, their spatial dependence is shown in the top frame. (b) Eigenfunction of the $n=100$ mode. 